\title{
Numerical Investigation of Wet Inflow in Steam Turbine Cascades Using NURBS-based Mesh Generation Method
}

\author{
Amir Ebrahimi-Fizik ${ }^{\text {a }}$ Esmail Lakzian ${ }^{\text {a* }}$, Ali Hashemian ${ }^{\text {b,c }}$ \\ a. Center of Computational Energy, Department of Mechanical Engineering, Hakim Sabzevari University, Sabzevar, Iran \\ b. BCAM - Basque Center for Applied Mathematics, Bilbao, Basque Country, Spain \\ c. Department of Mechanical Engineering, Hakim Sabzevari University, Sabzevar, Iran \\ * Corresponding author, email address: e.lakzian@hsu.ac.ir, Tel/Fax.: +98 5144012818
}

\begin{abstract}
In this paper, the impact of existence of wetness in the inflow of stationary cascades of steam turbine blades has been numerically investigated. A new mesh generation method based on non-uniform rational B-splines (NURBS) has been adopted to reduce the numerical error of the wet inflow simulation. Moreover, two common meshing scenarios namely blade-to-blade (B-B) and periodic-to-periodic boundary (P-P) are studied and different angle of the grid at the trailing edge have been considered. The classical nucleation theory corrected by CourtneyKantrowitz model and the Young's droplet growth model are employed to simulate the condensation phenomenon. By validating against experimental data, the results showed that implementing the proposed NURBS-based meshing technique decreased the prediction errors of static pressure distribution and droplet average radius by $35.64 \%$ and $78.44 \%$, respectively, in comparison to typical grid generation methods. In addition, it was observed that existence of wetness at inlet significantly decreased the supercooling degree and postponed the nucleation process. Thus, the nucleation rate could be ameliorated in the case when we have a specific amount of wetness fraction in the inflow.
\end{abstract}

Keyword: NURBS-based meshing technique; condensing flow; wet inflow

\section{Introduction}

Condensation, as a nonequilibrium and unsteady thermodynamic phenomenon, is commonly occurred in some engineering applications such as supersonic/transonic nozzles or the last stages of low-pressure steam turbines. Due to the destructive effects of condensation, this phenomenon has been investigated in many studies both numerically [1-8] and experimentally [9-13]. During the expansion of steam in the special condition in a low-pressure turbine blade, one-phase vapor changes into a two-phase mixture after supercooling and nucleation. The liquid phase of the mixture causes mechanical and thermodynamic losses and, as a result, reduces the efficiency at the last stages of low-pressure turbines. It is worth mentioning that the steam inflow is ideally assumed dry at the inlet of the stationary cascades. Whereas, because the upper rotor and stator blades are affected by the erosion damage, the flow may practically approach the stationary cascades as wet inflow. As many studies have been tried to model the dry inflow through stationary cascades, studying wet at inlet of the steam turbine cascades is essential. 


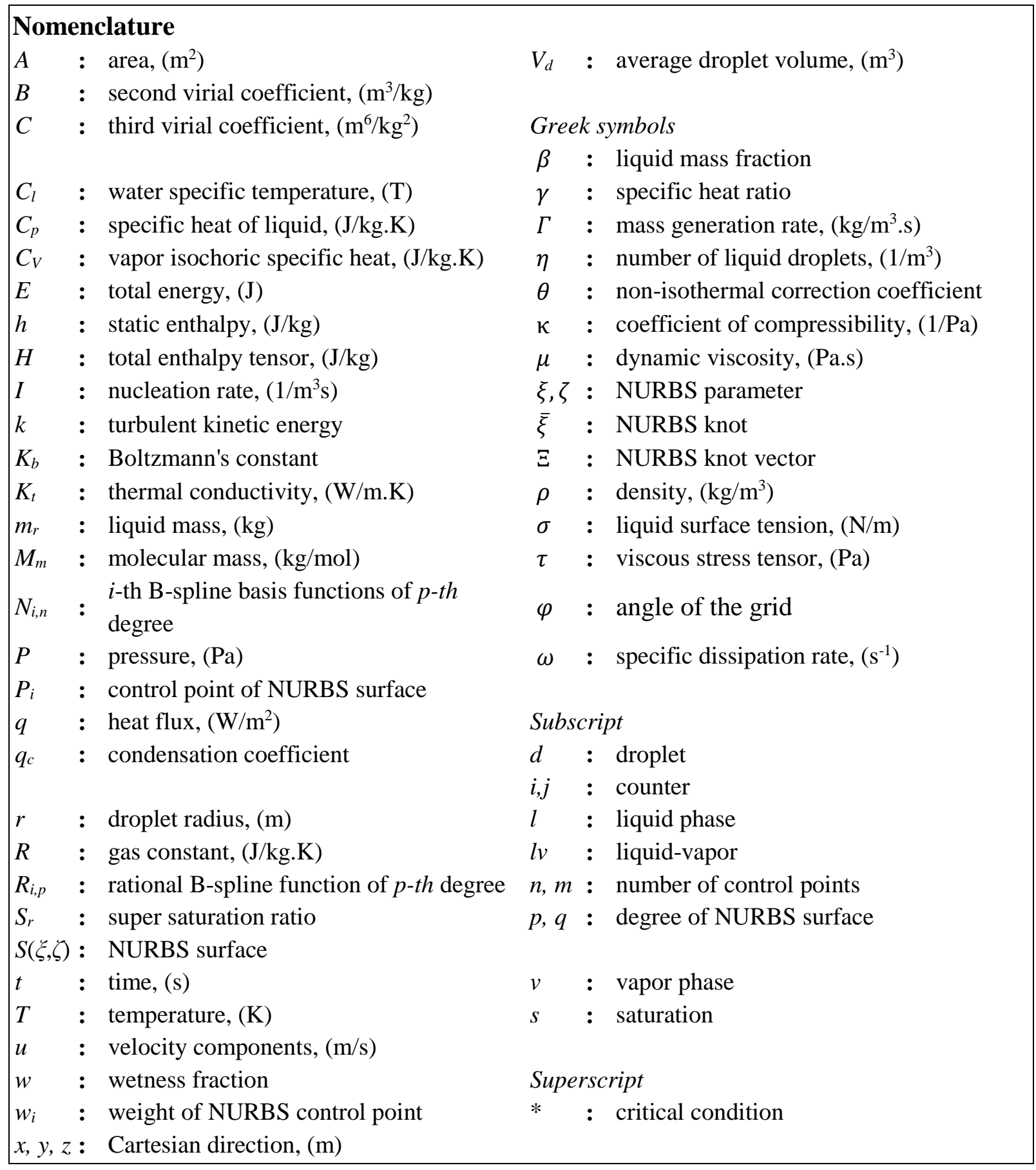

Different studies have been performed to offer solutions for improving the accuracy of modeling methods in steam turbine blades. In this regard, physical understandings of fluid and condensation phenomenon, along with implementing accurate numerical modeling methods are the main goals of these studies. For example, correcting nucleation equation, adopting more appropriate droplet growth model and considering the slip condition effects and coalescence are the most commonly offered solutions. Hagmeijer et al. [14] and Sinha et al. [15] employed the Hertz-Knudsen droplet growth model to investigate the wet-steam flow in a convergentdivergent nozzle. This model defines the droplet growth rate as the ratio of molecules' collision rate with the surface of droplets, to the evaporation rate from the droplets surface. Another 
model to calculate the droplet's growth rate was proposed by Young [16]. This model defines the droplet's growth as a function of evaporation and condensation coefficients. Also, a semiempirical relation was proposed by Bakhtar and Zidi [17] to calculate the droplet's growth rate. Based on this relation, the droplet's growth rate in the continuum and free molecular regimes is calculated by using the kinetic theory of gases. Lakzian and Masjedi [18] investigated the impacts of considering the slip condition between vapor and liquid phases on entropy generation in a condensing flow. According to their results, applying slip condition in computing causes a higher rate of entropy generation. Lakzian and Shaabani [19] modeled a condensing flow by considering the coalescence of formed droplets. They showed that taking into account the impacts of coalescence could decrease the rate of entropy generation and exergy losses.

Some other solutions, offered to achieve more accurate results, are applying more efficient mesh generation methods (e.g., O-mesh [20-22] or quasi grid [23, 24]) in order to reach a better agreement with experiments. Bakhtar and Tochai [25] investigated the effect of periodical outlet boundaries' angle in an inviscid wet-steam flow inside the passage between the two stationary cascades of blades by using a quasi-grid. However, most studies have been performed using the H-type grids. Mirhoseini and Boroomand [26] employed quadrilateral cells to investigate a multi-objective optimization of hot steam injection. Francesco et al. [27] studied the non-equilibrium condensation inside the cascades using a more efficient grid with a zero angle of the grid at the trailing edge and implementing a mixture model approach. Vatanmakan et al. [7] studied the effects of volumetric heating in the stationary cascades using an H-type grid and an angle of the grid of $64.4^{\circ}$.

Considering that reaching an analysis-suitable grid by using the above-mentioned conventional meshing techniques is generally a difficult and time-consuming process, especially when the flow passes through the free-form curved passage between cascades, the present paper proposed to employ the non-uniform rational B-spline (NURBS) formulation as a meshing technique for such geometries. Based on the fundamental properties of the NURBS formulation, the computational mesh with "curved" boundaries, which is well consistent with the geometry of the cascade, is inherently generated by geometric modeling of the flow passage, aiming at reducing the numerical simulation errors. Inasmuch as many real-world objects have been constructed by free-form curves and surfaces, the NURBS formulation appears to be a universal class for curve/surface modeling in computer-aided design, manufacture and engineering (CAD/CAM/CAE). The trajectory of a robot [28], geometry of a curved structure [29-31], and a multi-axis machining toolpath [32,33] are common examples modeled by NURBS formulation. In addition, NURBS has other advantages in comparison to polygonal meshing, e.g., faster generation, and lower processing load. A remarkable number of studies have been recently performed by using the NURBS formulation, e.g., for propellers [34, 35], optimum design of steam and wind turbine blades [36-38], gas-turbine blades [39] and airfoils [40,41]. The studies carried out using the NURBS technique have shown satisfactory agreement with experimental results.

In this paper, the effect of wetness at the inlet of steam turbine stationary cascades is investigated. We numerically show that the nucleation process could be ameliorated when we have a specific amount of wetness in the inflow. The main contribution of this work lies in the application of the NURBS formulation as a new mesh generation method in CFD problems, 
particularly those concerning the wet-steam flow simulation. The smooth and flexible geometry representation of the NURBS formulation not only improves the quality of the mesh generation, but also reduces the numerical error of the simulation. Using the NURBS-based meshing technique, we model two common meshing scenarios namely blade-to-blade (B-B) and periodic-to-periodic boundary (P-P). Additionally, we study the effect of considering different angles of the grid at the trailing edge (i.e., the angle between the horizontal axis and periodical outlet boundaries at downstream of the trailing edge). In the presented methodology, the Eulerian-Eulerian model, as well as the shear-stress transport (SST) $k$ - $\omega$ turbulence model is used to simulate the two-phase wet-steam flow in the last stages of a low-pressure steam turbine. Finally, the classical nucleation model corrected by Courtney [42] and Kantrowitz [43], and the Young's droplet growth model [44] is employed to model the condensation phenomenon.

\section{Mathematical modeling}

In the present study, the impacts of existence of wetness at the inlet of steam turbine stationary cascades are studied. In addition, the effect of using a NURBS-based mesh generation method with the aim of improving the mesh compatibility with the blade cascades is investigated. Then, by applying the generated mesh, a non-equilibrium and full turbulent condensing wet-steam flow is numerically modeled. The numerical model is based on Eulerian-Eulerian method with the SST $k-\omega$ turbulence model. For this simulation, the following assumptions are adopted:

- Wet-steam flow consists of continuous vapor phase (denoted by the subscript $v$ ) with a great number of liquid droplets (denoted by the subscript $l$ ).

- The droplets form is spherical.

- The droplets radius is $1 \mu m$ or less.

- The volume of condensed phase is negligible.

- The interaction between droplets is not taken into account since the interacting forces are directly related to the radius of the droplets, which is very small (see, e.g., $[3,45])$

- There is no slip condition between vapor and liquid phases since as studied in [46], the velocity of the droplets reaches to the vapor velocity almost immediately after the nucleation.

- The mass fraction of condensed phase is 0.2 or less (see, e.g., [47, 48]).

- The heat capacity of the fine droplet is negligible compared with the latent heat released during the condensation.

- The temperature and pressure of the vapor are applied to the corresponding amounts of the mixture.

- A set of two-dimensional viscous compressible Navier-Stokes equations are employed for the vapor phase.

- Two transport equations are used for the liquid phase mass fraction and the number of liquid droplets per unit volume [7].

In this regard, we review the governing equations of the flow as well as the equations representing the NURBS grid generation. As shown in Fig. 1, two different approaches are 
considered to simulate and investigate the wet-steam flow. The first approach (see Fig. 1a) simulates the flow inside the passage between two stationary cascades of blades, designated as B-B (blade-to-blade) approach. The second models the wet-steam flow around a stationary blade, from midline to midline denoted by P-P (periodic-to-periodic boundary) approach, as shown in Fig. 1b. Moreover, the angle of the grid $(\varphi)$ defined as the angle between $x$-axis and periodical outlet boundaries at downstream of the trailing edge is shown in the figure.

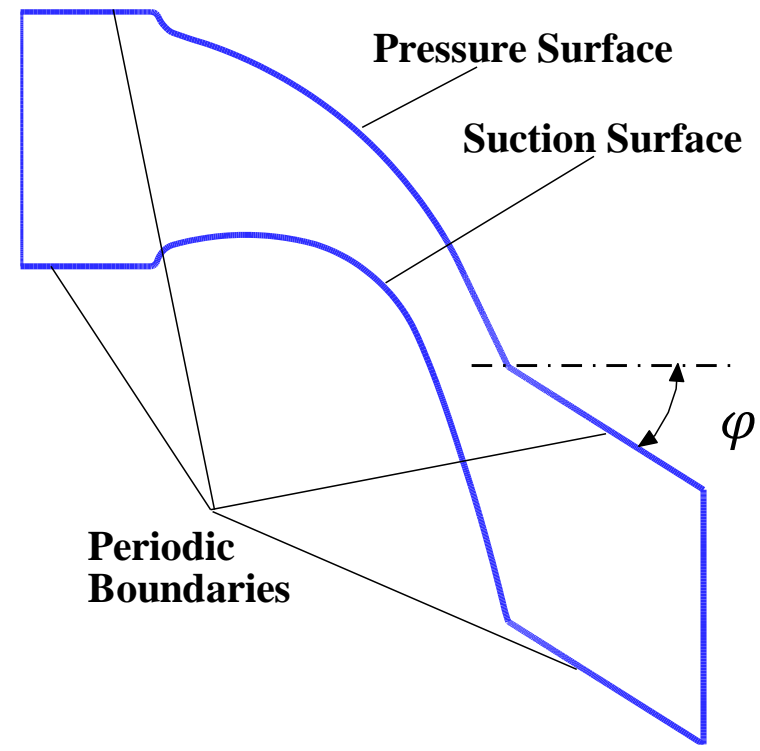

a

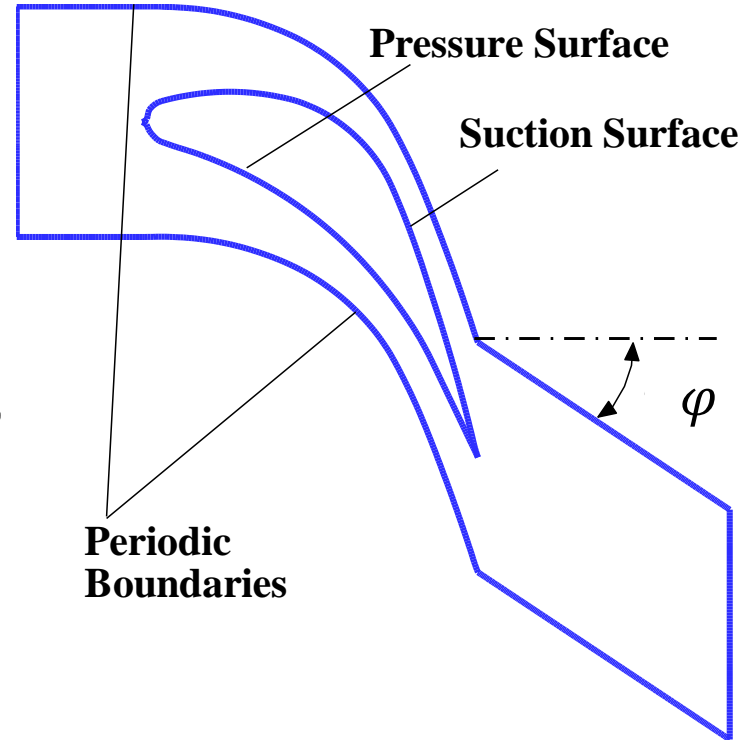

b

Fig. 1: Computational domain a) inside the passage between two cascades from blade to blade (B-B), b) around a cascade from periodic boundary to periodic boundary (P-P).

\subsection{Conservation equations}

Considering a compressible flow, the conservation of mass, momentum, and energy equations could be defined as follows [10]:

$$
\begin{aligned}
& \frac{\partial \rho}{\partial t}+\frac{\partial}{\partial x_{j}}\left(\rho u_{j}\right)=0 \\
& \frac{\partial}{\partial t}\left(\rho u_{j}\right)+\frac{\partial}{\partial x_{j}}\left(\rho u_{i} u_{j}\right)=-\frac{\partial p}{\partial x_{j}}+\frac{\partial \tau_{i j}}{\partial x_{j}} \\
& \frac{\partial}{\partial t}(\rho H)+\frac{\partial}{\partial x_{j}}\left(\rho u_{j} H\right)=\frac{\partial q_{j}}{\partial x_{j}}+\frac{\partial}{\partial x_{j}}\left(u_{i} \tau_{i j}\right)
\end{aligned}
$$

where, the viscous stress tensor $\tau_{i j}$, heat flux vector $q_{i}$ and total enthalpy $H$, are given by:

$$
\begin{aligned}
\tau_{i j} & =\mu\left(\frac{\partial u_{i}}{\partial x_{j}}+\frac{\partial u_{j}}{\partial x_{i}}\right) \\
q_{i} & =-\kappa \frac{\partial T}{\partial x_{j}} \\
H & =h+\frac{1}{2} u_{i} u_{i}
\end{aligned}
$$

and $u_{i}$ and $u_{j}$ are the velocity components. In addition, $p, \tau, \kappa$, and $q$ correspond to pressure, viscous stress tensor, coefficient of compressibility, and heat flux, respectively. Finally, $\rho$ is the density of the mixture that is related to the density of the vapor phase as follows: 


$$
\rho=\frac{\rho_{v}}{(1-\beta)}
$$

In the above equation, $\beta$ denotes the mass fraction of liquid phase. The conservation equations are formulated with regard to the properties of two-phase mixture which can be estimated by the following equation [5]:

$$
\phi_{\text {mix }}=\phi_{l} \beta+(1-\beta) \phi_{v}
$$

where $\phi$ represents the properties such as enthalpy $(h)$, specific heat $\left(C_{p}\right.$ and $\left.C_{v}\right)$, dynamic viscosity $(\mu)$, and thermal conductivity $\left(K_{t}\right)$.

Two additional transport equations are required in order to model the wet-steam condensation flow. The first equation is related to the mass fraction of the liquid phase designated as $\beta$ (eq. 5).

$$
\frac{\partial \rho}{\partial t} \beta+\frac{\partial}{\partial x_{i}}\left(\rho u_{i} \beta\right)=\Gamma
$$

where $\Gamma$ is defined as the mass generation rate in $\mathrm{kg}$ per unit of volume per second which is due to condensation and evaporation. The second transport equation estimates the number of liquid droplet per unit of volume designated as $\eta$ (eq. 6).

$$
\frac{\partial \rho}{\partial t} \eta+\frac{\partial}{\partial x_{i}}(\rho \eta \beta)=\rho I
$$

where $I$ shows nucleation rate in number of generated droplets per unit of volume per second and $\rho$ is the density of the humid vapor. Moreover, the number of droplets, $\eta$, is defined as:

$$
\eta=\frac{\beta}{(1-\beta) V_{d}\left(\rho_{l} / \rho_{v}\right)}
$$

where $V_{d}$ denote the average droplet volume that is calculated based on average droplet radius, $\bar{r}_{d}[49]$.

$$
V_{d}=\frac{4}{3} \pi \bar{r}_{d}^{3}
$$

\subsection{Nucleation rate and droplet growth models}

The condensation phenomenon involves two major processes of nucleation and droplet growth. The classical nucleation theory presents the nucleation and formation of spherical droplets from a supersaturated condition. Based on this theory, a steady-state homogenous nucleation rate is formulated as follows [7]:

$$
I_{\text {classic }}=q_{c} \frac{\rho_{v}^{2}}{\rho_{L}} \sqrt{\frac{2 \sigma}{\pi M_{m}^{3}}} \exp \left(-\frac{4 \pi r^{* 2} \sigma}{3 k_{b} T_{v}}\right)
$$

where $q_{c}, k_{b}, M_{m}$, and $\sigma$ are, respectively, the condensation coefficient, the Boltzmann constant, the mass of one molecule and liquid surface tension and $r^{*}$ is the Kelvin-Helmholtz critical droplet radius which is given by [50]:

$$
r^{*}=\frac{2 \sigma}{\rho_{l} R T \ln S_{r}}
$$

where $S_{r}$ is the supersaturation ratio defined as the ratio of the vapor pressure to the equilibrium saturation pressure. Since the expansion of vapor is occurred rapidly, when the state path passes 
through the saturation line, the process changes into non-equilibrium condition. Therefore the supersaturation ratio can be greater than one [7].

$$
S_{r}=\frac{P}{P_{S}(T)}
$$

A variety of corrections is proposed to apply to classic nucleation theory for non-isothermal homogeneous condensation. Among them, the accuracy of the Courtney-Kantrowitz correction has been proven by previous studies (see, e.g., [24, 51, 52]).

$$
I=\frac{1}{(1+\theta)} I_{\text {classic }}
$$

In the above equation, $\theta$ denotes the non-isothermal correction defined by the following equation:

$$
\theta=2 \frac{\gamma-1}{\gamma+1}\left(\frac{h_{l v}}{R T}\right)\left(\frac{h_{l v}}{R T}-\frac{1}{2}\right)
$$

where $h_{l v}$, and $\gamma$ are the enthalpy of evaporation and the specific heat ratio $(\approx 1.32)$, respectively [53]. Based on the classical nucleation theory, the mass generation rate is defined as the summation of mass increase caused by nucleation and also due to growth of droplets [49].

$$
\Gamma=\frac{4}{3} \pi \rho_{l} I r^{* 3}+4 \pi \rho_{l} \eta \bar{r}^{2} \frac{\partial \bar{r}}{\partial t}
$$

where $\partial \bar{r} / \partial t$ is the droplet growth rate written as follows [16]:

$\frac{\partial \bar{r}}{\partial t}=\frac{P}{h_{l v} \rho_{l} \sqrt{2 \pi R T}} \frac{\gamma+1}{2 \gamma} C_{P}\left(T_{l}-T_{v}\right)$

The following relation was proposed to determine the droplet temperature in terms of vapor temperature, supercooling degree and droplet radius [54]:

$$
T_{l}=T_{s}(p)-\left[T_{s}(p)-T_{v}\right] \frac{r^{*}}{r}
$$

It should be noted that the droplet radius is assumed to be $1 \mu \mathrm{m}$ or less.

\subsection{Equation of state}

The relation between pressure, temperature and density of the vapor is expressed in terms of the equation of state as follows:

$$
P=\rho_{v} R T\left(1+B \rho_{v}+C \rho_{v}^{2}\right)
$$

where $B$, and $C$ are the second and third virial coefficients (given in $\mathrm{m}^{3} / \mathrm{kg}$ and $\mathrm{m}^{6} / \mathrm{kg}^{2}$ respectively) defined as follows [7]:

$$
\begin{aligned}
& B=b_{1}\left(1+\frac{\tau_{B}}{\alpha_{B}}\right)^{-1}+b_{2} e^{\tau_{B}}\left(1-e^{-\tau_{B}}\right)^{5 / 2}+b_{3} \tau_{B} \\
& C=c_{1}\left(\tau_{C}-\tau_{0}\right) e^{-(\alpha \tau)_{C}}+c_{2}
\end{aligned}
$$

It should be noted that $\tau_{B}=1500 / T, \tau_{C}=T / 647.286, \tau_{0}=0.89780, \alpha_{B}=10000$, $\alpha_{C}=11.16, \quad b_{1}=0.0015, \quad b_{2}=0.000942, \quad b_{3}=-0.0004882, \quad c_{1}=1.722, \quad$ and 
$c_{2}=1.5 \times 10^{-6}$. In addition, the two above equations are expressed only for the temperature range from 273 to 1073 degrees [55].

\subsection{Turbulence modeling}

In this paper, the SST $k-\omega$ model is employed to simulate the turbulent wet-steam flow inside the passage between two stationary cascades of blades. It should be noted the mentioned model was developed by Menter [56] to simulate more effectively in both regions near the wall and the far field. Therefore, it is more accurate and more reliable for transonic shock waves flows than the other models $[7,57,58]$. Equations for turbulent kinetic energy, $k$, and specific dissipation rate, $\omega$, are as follows [56]:

$$
\begin{aligned}
& \frac{\partial}{\partial t}(\rho k)+\frac{\partial}{\partial x_{i}}\left(\rho k u_{i}\right)=\frac{\partial}{\partial x_{j}}\left(\Gamma_{k} \frac{\partial k}{\partial x_{j}}\right)+\widetilde{G}_{k}-Y_{k}+S_{k} \\
& \frac{\partial}{\partial t}(\rho \omega)+\frac{\partial}{\partial x_{i}}\left(\rho \omega u_{i}\right)=\frac{\partial}{\partial x_{j}}\left(\Gamma_{\omega} \frac{\partial \omega}{\partial x_{j}}\right)+G_{\omega}-Y_{\omega}+D_{\omega}+S_{\omega}
\end{aligned}
$$

where $\tilde{G}_{k}$, and $G_{\omega}$, respectively, represent the generation of turbulence kinetic energy due to medium velocity gradients and the generation of $\omega$, and $Y_{k}$, and $Y_{\omega}$ denote the dissipation of $k$, and $\omega$. Additionally, $S_{k}$, and $S_{\omega}$ are user-defined source terms, $D_{\omega}$ is the cross-diffusion term, and $\Gamma_{k}$, and $\Gamma_{\omega}$ are defined as the effective diffusivity of $k$, and $\omega$, respectively. Further details on the calculation of mentioned terms are described in [56].

\subsection{NURBS grid generation}

In the present paper, the NURBS formulation has been employed for generating an appropriate grid that is more compatible with the geometry of the turbine cascade. More precisely, the physical domain of the fluid flow through the passage can be interpreted by a planar NURBS surface as a mapping from the bivariate parameter space $(\xi, \zeta) \in[0,1]^{2}$ onto the geometric (i.e. physical) space $S(\xi, \zeta)=[x(\xi, \zeta), y(\xi, \zeta)]$. This NURBS surface of degree $(p, q)$ is characterized by a bidirectional net of $(n+1)(m+1)$ control points $P_{i j}=\left[P_{x}^{i j}, P_{y}^{i j}\right]$ and respective weights $w_{i j}$ as:

$$
S(\xi, \zeta)=\sum_{j=0}^{m} \sum_{i=0}^{n} R_{i j}(\xi, \zeta) P_{i j}
$$

where $R_{i j}(\xi, \zeta)$ is the piecewise rational basis function given by:

$$
R_{i j}(\xi, \zeta)=\frac{N_{i, p}(\xi) N_{j, q}(\zeta) w_{i j}}{\sum_{k=0}^{n} \sum_{l=0}^{m} N_{k, p}(\xi) N_{l, q}(\zeta) w_{k l}}
$$

and $N_{i, p}(\xi)$ and $N_{j, q}(\zeta)$ are the $i$-th and $j$-th B-spline basis functions of degree $p$ and $q$, respectively, defined by the Cox-de Boor recursion formula over the non-decreasing knot vectors $\Xi$ and $\mathrm{Z}$ as follows [59].

$$
\begin{aligned}
& \Xi=[\underbrace{0,0, \cdots, 0}_{p+1}, \xi_{p+1}, \xi_{p+2}, \cdots, \xi_{n}, \underbrace{1,1, \cdots, 1}_{p+1}] \\
& \mathrm{Z}=[\underbrace{0,0, \cdots, 0}_{q+1}, \zeta_{q+1}, \zeta_{q+2}, \cdots, \zeta_{m}, \underbrace{1,1, \cdots, 1}_{q+1}]
\end{aligned}
$$




$$
\begin{aligned}
N_{i, 0}(\xi) & = \begin{cases}1 & \xi_{i} \leq \xi<\xi_{i+1} \\
0 & \text { otherwise }\end{cases} \\
N_{i, p}(\xi) & =\frac{\xi-\xi_{i}}{\xi_{i+p}-\xi_{i}} N_{i, p-1}(\xi)+\frac{\xi_{i+p+1}-\xi}{\xi_{i+p+1}-\xi_{i+1}} N_{i+1, p-1}(\xi)
\end{aligned}
$$

Fig. 2 demonstrates a bicubic (i.e., $p=q=3$ ) surface as a mapping from the parameter space to the physical space. Based on the different increments of parameters $\xi$ and $\zeta$ on the parameter space, we can reach different mesh densities on the geometric domain. These "curved" grids can then be considered as the computational mesh of the fluid flow for further analysis. The more interesting advantage of this meshing technique is that we can easily import degrees, control points, weights and knot vectors from the CAD model of the cascade, so that the grid is inherently generated without requiring any additional task.

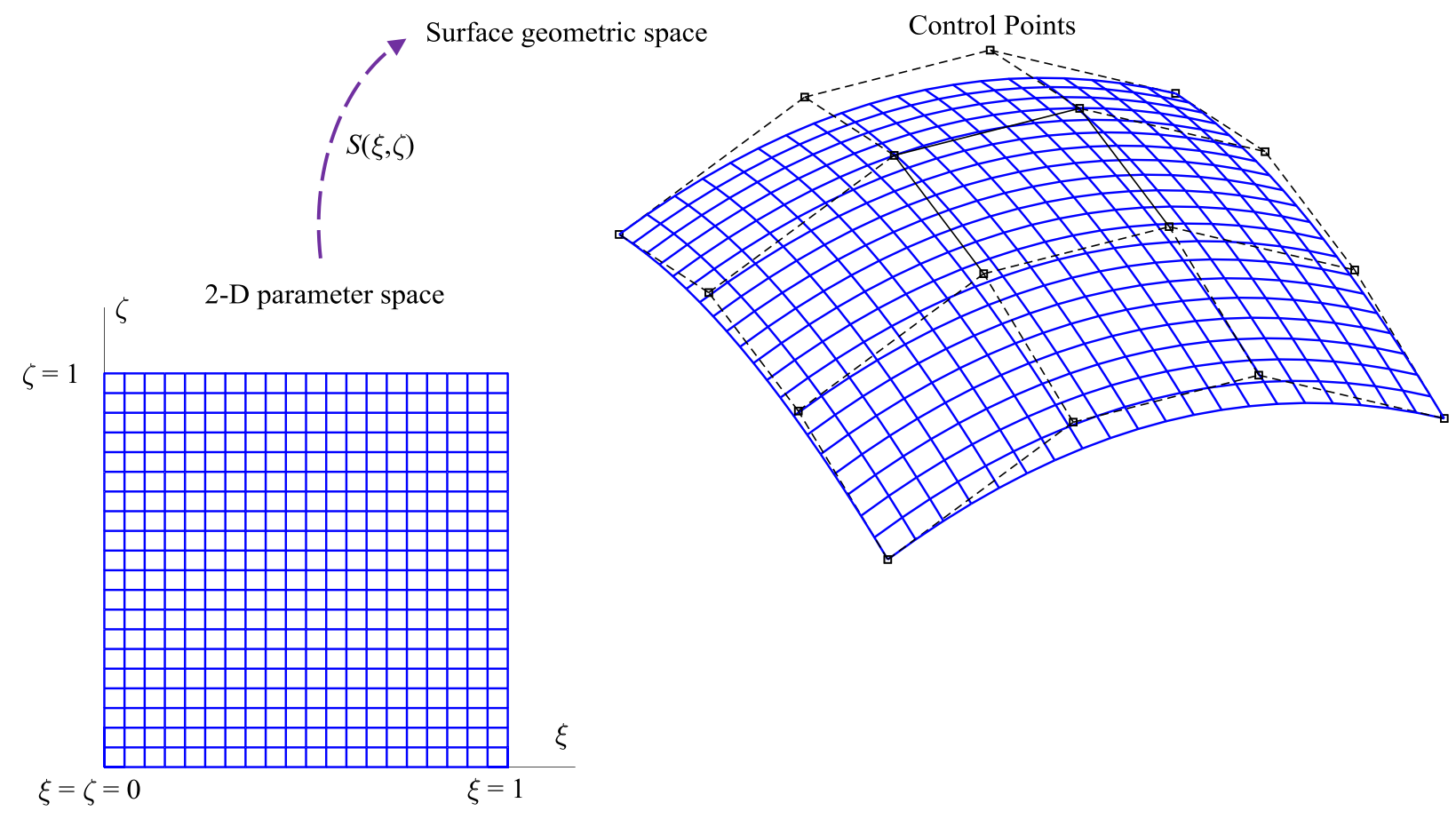

Fig. 2: A bicubic surface in the physical space (right) and its corresponding domain in the parameter space (left)

\section{Numerical method}

In this paper, the NURBS surface modeling was used to generate the appropriate mesh in computational domain of both proposed B-B and P-P approaches with different values of angle of the grid, $\varphi$. The 2D Reynolds-averaged Navier-Stokes (RANS) equations were used for numerical simulation in the compressible, viscous, turbulent, and steady state flow. In this regard, an implicit density based Eulerian-Eulerian approach was employed by using the SST $k-\omega$ model. Also, the Roe's method [60] was implemented to compute the convective fluxes. The conservation equation discretized by using the upwind scheme of second-order accuracy in space. In this scheme, the face property value $\phi$ is computed using the following expression:

$$
\phi_{i+1}=\phi_{i}+\phi_{i}^{*} r_{i}
$$

where $\phi, \phi_{i}^{*}$, and $r_{i}$ are, respectively, the cell-centered value in the upstream cell, the correction coefficient, and the distance between upstream cell center and the cell face center. The value of $\phi_{i}^{*}$ can be defined as follows [61]: 


$$
\phi_{i}^{*}=\frac{1}{V} \sum_{f}^{N_{f}} \tilde{\phi}_{i} A_{i}
$$

where $\tilde{\phi}_{i}$ and $N_{f}$ are the average value of the two cells adjacent to the face and the number of nodes on the face, respectively.

The solutions converged to normalized RMS residuals in the order of $10^{-6}$ or less. Geometrical specifications of the turbine blade profile are presented in Table 1. In addition, the boundary conditions at inlet and outlet are given in Table 2 . It should be mentioned that the outflow is assumed as a supersonic flow.

Table 1: Geometrical specifications of the blade profile

\begin{tabular}{lllll}
\hline Length $(\mathbf{m m})$ & Chord $(\mathbf{m m})$ & Pitch $(\mathbf{m m})$ & Axial chord $(\mathbf{m m})$ & Inlet flow angle \\
\hline 76.00 & 35.76 & 18.26 & 25.27 & 0 \\
\hline
\end{tabular}

Table 2: Boundary conditions of the turbine blade cascade

\begin{tabular}{ll}
\hline $\boldsymbol{P}_{\mathbf{0}, \text { in }}(\mathrm{kPa})$ & 172 \\
$\boldsymbol{T}_{\mathbf{0}, \text { in }}=\boldsymbol{T}_{\boldsymbol{s}}\left(\boldsymbol{P}_{\mathbf{0}, \text { in }}\right)-\mathbf{8}(\mathrm{K})$ & 380.66 \\
$\boldsymbol{P}_{\text {out }}=\mathbf{0 . 4 8} \boldsymbol{P}_{\mathbf{0}, \text { in }}(\mathrm{kPa})$ & 82.56 \\
\hline
\end{tabular}

\section{Results}

\subsection{Validation}

As shown in Fig. 3, in order to achieve a suitable value of angle of the grid $\varphi$, three different values were considered. The first considered value of $\varphi$ is selected as $0^{\circ}$ which is investigated in many studies (see, e.g., Francesco et al. [27], Liberson and Hesler [62], and Rueda et al. [63]). The second value of $\varphi$ is chosen as $64.4^{\circ}$ so that the outlet periodic boundary is tangent to the pressure surface which has been suggested by some studies in the literature (see, e.g., Bkhtar et al. [25, 52] and Vatanmakan et al. [7]). Moreover, the average quantity of mentioned angles was determined as the third value of $\varphi$. It should be the mentioned that every grid consists of three parts namely inlet periodical section, passage section, and outlet periodical section. In order to generate the NURBS-based grids in each part, different numbers of control points ( $m$ and $n$ ) and surface degrees ( $p$ and $q$ ) are needed which were selected based on the complexity of the geometry and provided CAD data of the blades. Therefore, the applied values of $m, n, p$, and $q$ at each part are shown in Table 3. In addition, we perform a grid dependence study for both B-B and P-P meshing approaches (see Fig. 4). However, since the convergence rates for different angles of the grid are very close, herein only the case of $\varphi=64.4^{\circ}$ is reported and the specification of the independent mesh of other grids is described in Table 4. It is important to note that since in the B-B grid, the computational domain is considered between the suction and pressure sides of two consecutive blades (i.e., the blade walls are the outer boundary conditions), the accuracy of the results near the walls is subject to a lower numerical error and we can consider almost the same mesh density everywhere. This is a true assumption as already investigated in, e.g., [23, 64]. On the other hand, in the P-P approach, the 
a

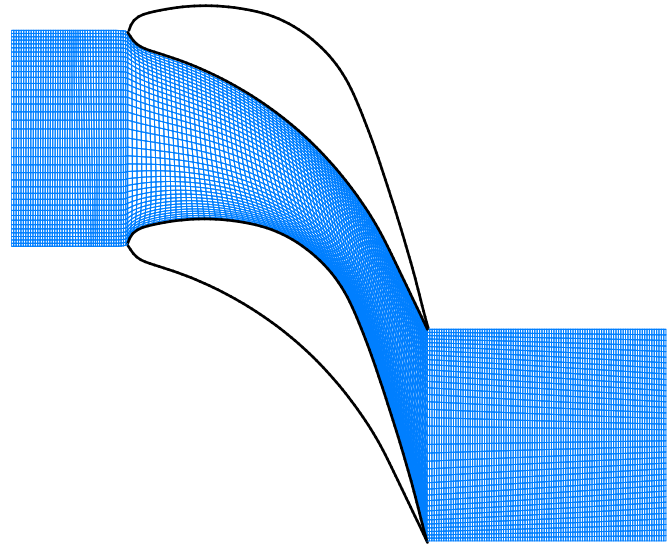

b

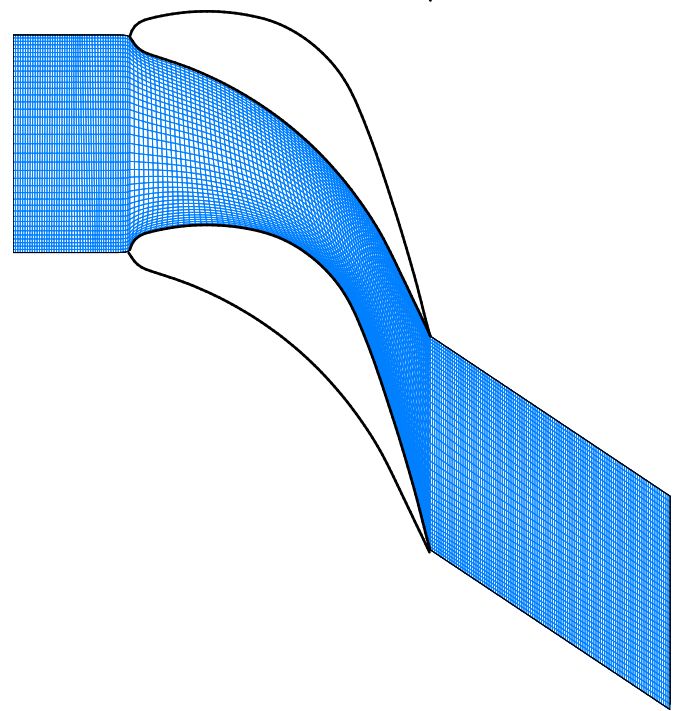

c

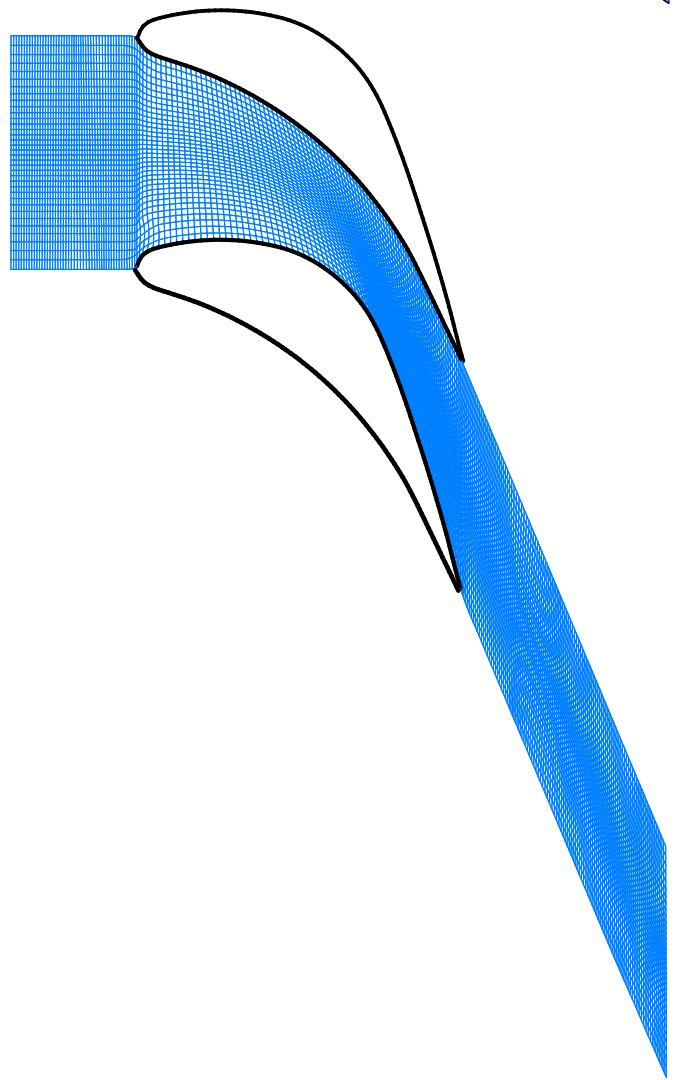

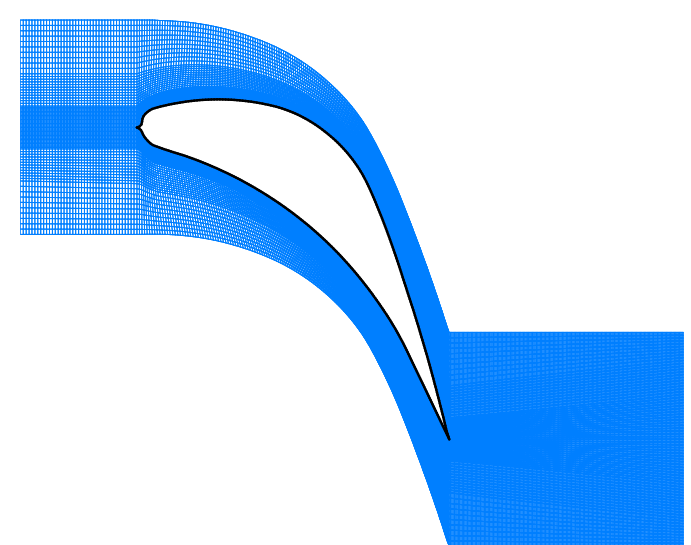
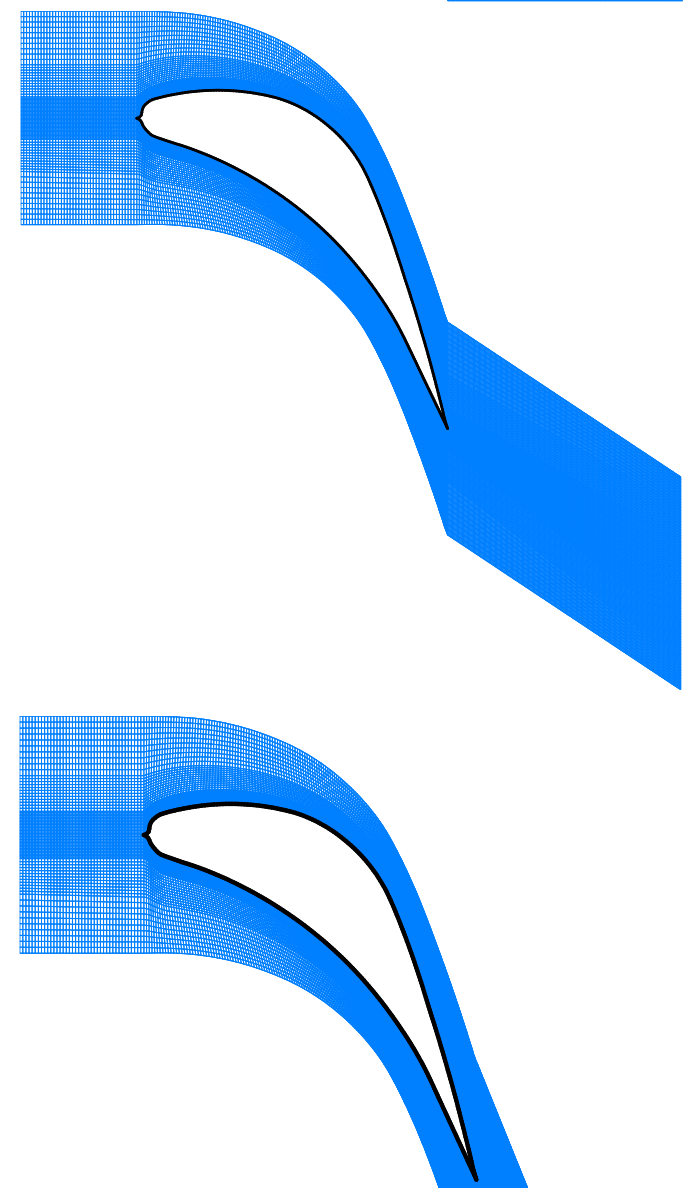

Fig. 3: Computational domain of B-B (left) and P-P (right) approaches with different values of $\varphi$ for steam turbine blade cascade, a) $\varphi=0$, b) $\varphi=32.2^{\circ}$, and c) $\varphi=64.4^{\circ}$ 
computational domain is considered from the periodic boundary (acting as outer boundary conditions) to the wall boundary on the suction side of the blade, and then from the wall boundary on the pressure side up to the periodic boundary. This entails a more complicated domain compared to the B-B approach and, therefore, we need a higher mesh density to improve the accuracy near the walls (see, e.g., [4]).

To validate the accuracy of the numerical modeling and investigate the effect of applying the NURBS-based mesh generation technique, the numerical results of all proposed grids were compared to experimental data conducted by Bakhtar et al. [23]. As seen in Fig. 4, the calculated results are well consistent with the experimental data and the location of shock is predicted with sufficient accuracy. Additionally, according to Fig. 5b, the estimation of the quantity of droplet average radius is improved compared to the conventional H-type grid's result. This blade-to-blade grid with a $30 \times 207$ mesh is presented in [23].

Table 3: The specification of the NURBS-based generated grids

\begin{tabular}{c|c|c|c|c|c|c}
\hline Section & \multicolumn{2}{|c|}{ Inlet periodical } & \multicolumn{2}{|c|}{ Passage } & \multicolumn{2}{c}{ Outlet periodical } \\
\hline Approach & B-B & P-P & B-B & \multicolumn{2}{c|}{ P-P } & B-B \\
\hline $\boldsymbol{m}$ & 19 & 2 & 57 & $\begin{array}{c}89 \text { (upside the blade) } \\
60 \text { (downside the blade) }\end{array}$ & 25 & $\begin{array}{c}7 \text { (upside the trailing edge) } \\
11 \text { (downside trailing edge) }\end{array}$ \\
$\boldsymbol{n}$ & 2 & 2 & 10 & 2 & 10 & 2 \\
$\boldsymbol{p}$ & 1 & 1 & 3 & 1 & 3 & 3 \\
$\boldsymbol{q}$ & 3 & 1 & 3 & 3 & 3 \\
\hline
\end{tabular}
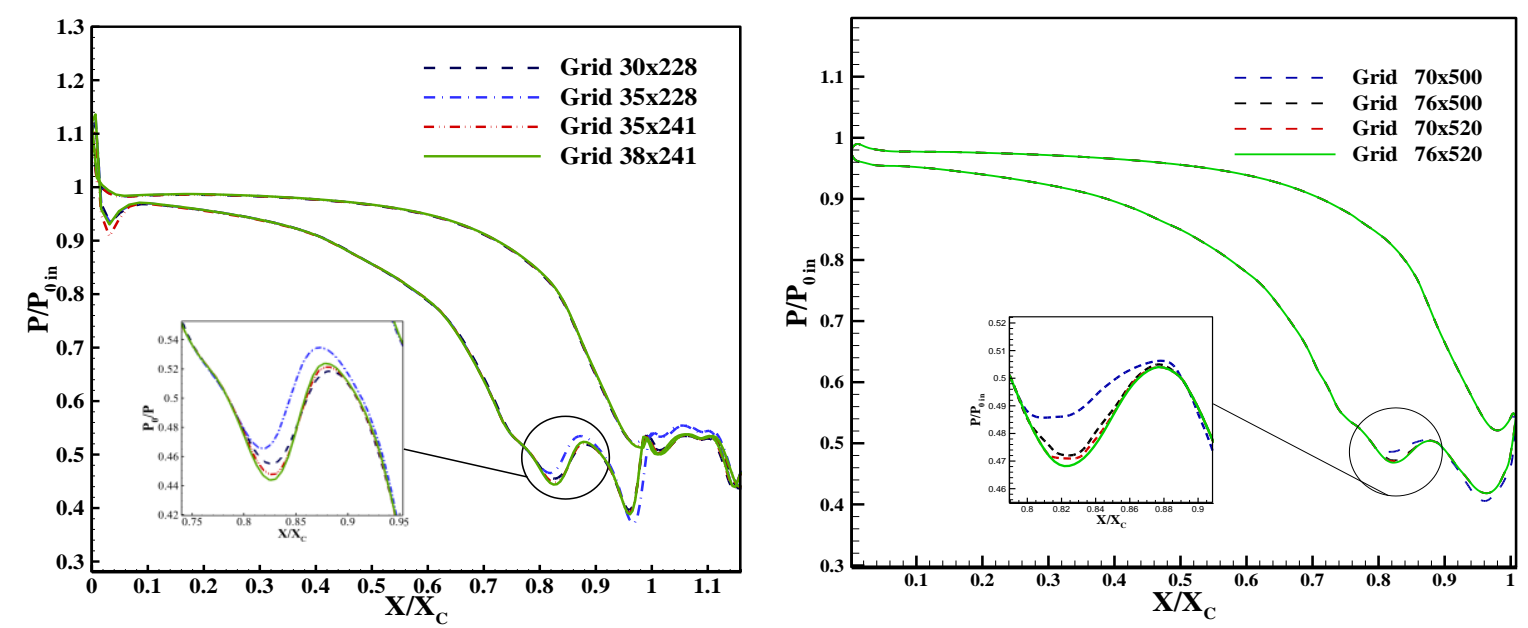

Fig. 4: Grid dependence results for B-B (left) and P-P (right) meshing approaches with $\varphi=64.4^{\circ}$

Table 4: Optimum grids for each proposed grid

\begin{tabular}{ccc}
\hline $\boldsymbol{\varphi}\left({ }^{\circ}\right)$ & B-B approach & P-P approach \\
\hline $\mathbf{0 0 . 0}$ & $35^{*} 248$ (Fig. 3a, Left) & $76^{* 410}$ (Fig. 3a, Right) \\
$\mathbf{3 2 . 2}$ & $35^{*} 248$ (Fig. 3b, Left) & $76^{* 420}$ (Fig. 3b, Right) \\
$\mathbf{6 4 . 4}$ & $38^{*} 241$ (Fig. 3c, Left) & $76 * 520$ (Fig. 3c, Right) \\
\hline
\end{tabular}




\subsection{Numerical results improvement by employing NURBS grid}

Distribution of static pressure in the proposed grids of B-B approach is shown by Fig. 5a. As it is indicated, the numerical results for each value of $\varphi$ show a satisfactory agreement with the experimental data. According to Table 5, it can be seen that the minimum error of B-B approach on the pressure side, suction side, and outlet periodical boundaries for different values of $\varphi$ occur with $3.17 \%$ at $64.4^{\circ}, 5.22 \%$ at $32.2^{\circ}$, and $0.55 \%$ at $64.4^{\circ}$, respectively (See Table 5). Due to the fact that the expansion rate has higher values on the suction side, nucleation is begun earlier and, as a result, the shock has more impact on the suction surface. However, the prediction accuracy of other boundaries could not be ignored. Therefore, the grid with an angle of the grid of $64.4^{\circ}$ is selected as the optimum grid of the B-B approach based on the minimum average error $(2.98 \%)$.

Fig. $5 \mathrm{~b}$ represents the distribution of static pressure for different quantities of angle of the grid in the P-P approach. As shown in this figure, the calculated results of all grids with various values of $\varphi$, show an adequate agreement with experimental data. The minimum error of P-P approach's grids on pressure and the suction side, and outlet periodical boundaries are related to the grids with the angle of the grid of $64.4^{\circ}$ by $2.71 \%, 32.2^{\circ}$ by 5.37 , and $64.4^{\circ}$ by $1.55 \%$, respectively (see Table 5). Therefore, the grid with the average error of $3.23 \%$ is proposed as the optimal grid $\left(\varphi=64.4^{\circ}\right)$ of P-P approach. Regarding Table 5, the proposed grid of the B-B approach $\left(\varphi=64.4^{\circ}\right)$ with the average error of $2.98 \%$ compared to the proposed one of the P-P approach of the same angle of the grid with the average error of 3.23\%, has better agreements with experimental data. Therefore, the B-B approach's domain with an angle of the grid of $64.4^{\circ}$ is finally proposed and selected to compare with the conventional H-type grid.

In order to figure out the impact of applying the NURBS technique, in addition to the experimental data, the proposed grid was compared with a grid generated by the conventional $\mathrm{H}$-type meshing method. In this regard, the error of computed results of the H-type grid, given by Bakhtar et al. [23], is represented in Table 5 .

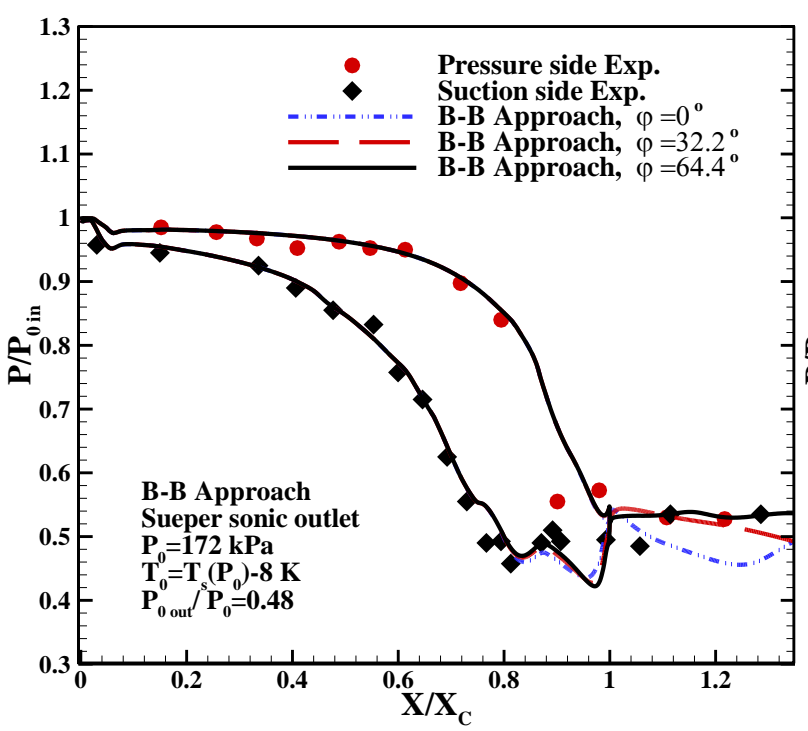

a

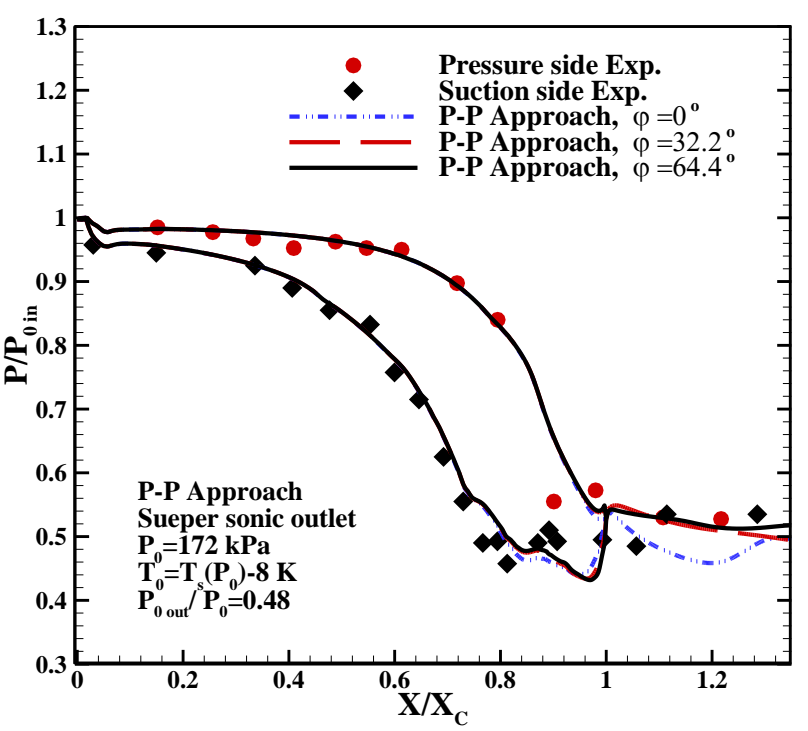

b

Fig. 5: Comparison of the static pressure distribution calculated by a) B-B, b) P-P approach with experimental data 
Table 5: Error of pressure distribution in different values of $\varphi$ by implementing B-B approach, P-P approach, and H-type grid [23] (scaled in \%)

\begin{tabular}{lccccccc}
\hline \multicolumn{1}{c}{ approach } & \multicolumn{3}{c}{ B-B } & & & P-P & H-type grid [23] \\
\hline \multicolumn{1}{c}{$\boldsymbol{\varphi}\left({ }^{\circ}\right)$} & $\mathbf{0}$ & $\mathbf{3 2 . 2}$ & $\mathbf{6 4 . 4}$ & $\mathbf{0}$ & $\mathbf{3 2 . 2}$ & $\mathbf{6 4 . 4}$ & - \\
\hline Pressure Side & 3.21 & 3.18 & 3.17 & 2.86 & 2.74 & 2.71 & 0.80 \\
Suction Side & 5.25 & 5.22 & 5.24 & 5.4 & 5.37 & 5.42 & 5.74 \\
Periodical Boundary at outlet & 8.81 & 1.54 & 0.55 & 9.83 & 2.59 & 1.55 & 7.34 \\
Average Error & 5.76 & 3.31 & 2.98 & 6.03 & 3.57 & 3.23 & 4.63 \\
\hline
\end{tabular}

The comparison of the static pressure distributions of the proposed NURBS-based and Htype grids with experimental data is represented by Fig. 6a. As can be seen, the proposed grid generated based on NURBS method, tracked and predicted the shock closer. Additionally, according to Table 5, the H-type grid resulted in a lower error only on the pressure side by the value of $0.80 \%$ compared to $2.71 \%$ of the B-B approach NURBS grid. However, the results of the proposed grid generation method showed more accuracy in predicting the location of shock and the static pressure distribution on the suction surface and periodical boundaries at the outlet. In addition, the average error of the B-B approach is lower than the H-type grid with an improvement of approximately $1.65 \%$. Moreover, it can be observed that the proposed NURBS grid could carefully capture the two pressure increases at downstream of the trailing edge that is due to the shock wave at that region. The first rise is the inflection of the pressure surface shock on the suction side and the second one is originated from the suction surface (Fig. 6a and Fig. 8a).

The variation in the droplet average radius on the suction and pressure sides of both proposed (B-B approach of $\varphi=64.4^{\circ}$ ) and the H-type grids is represented in Fig. 6b. It can be inferred that the prediction of the droplet average radius at the outlet of the blade cascade is improved by applying the NURBS-based grid generation technique. Comparison to the H-type grid, the proposed NURBS grid decreased the error of droplet radius prediction by $15.70 \%$ and $14.94 \%$ on suction and pressure sides, respectively.

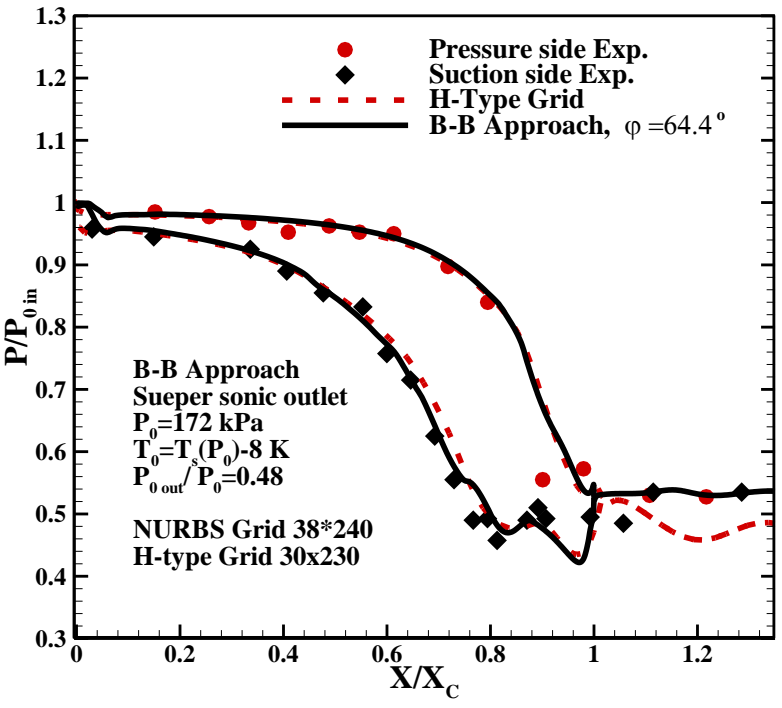

a

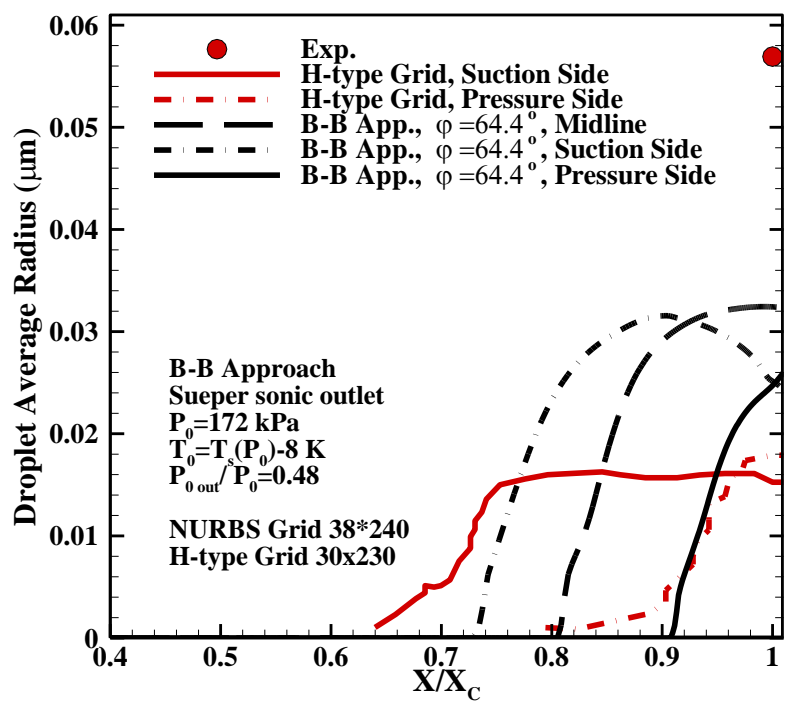

b

Fig. 6: Comparison of calculated results by the proposed grid with H-type grid's result and experimental data [23], a) static pressure distribution and b) droplet average radius 


\subsection{Effects of wet at inlet on the condensation phenomenon}

The schematic of wet inflow at the inlet of the stationary cascade of the steam turbine is illustrated by Fig. 7. It should be noted that the inflow involves numbers of fine spherical droplets. In order to investigate the impacts of wet at inlet, wet-steam inflow with different characteristics has been considered which are given in detail by Table 6 .

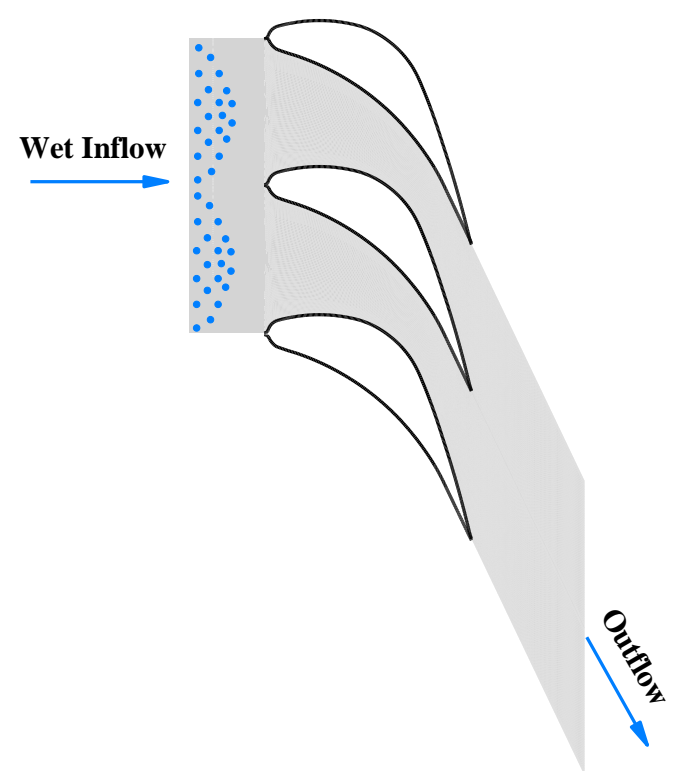

Fig. 7: Schematic of wet at the inlet of the stationary cascade of the steam turbine blade

Table 6: The characteristics of wet steam inflows inside the passage

\begin{tabular}{cccc}
\hline Case & Droplet Average Radius $(\boldsymbol{\mu m})$ & Average Wetness Fraction $(\%)$ & Number of Droplets \\
\hline 1 & 0.000 & 0.0 & 0 \\
2 & 0.111 & 0.5 & $1 \times 10^{15}$ \\
3 & 0.139 & 1.0 & $1 \times 10^{15}$ \\
4 & 0.158 & 1.5 & $1 \times 10^{15}$ \\
5 & 0.172 & 2.0 & $1 \times 10^{15}$ \\
6 & 0.188 & 2.5 & $1 \times 10^{15}$ \\
7 & 0.197 & 3.0 & $1 \times 10^{15}$ \\
\hline
\end{tabular}

The distribution of the static pressure is shown in Fig. 8. Considering a slight amount of wetness at the inlet, significant influences on the condensation zone and the pressure drop are observed. Fig. 8a shows that the condensation occurs at $x / x_{c} \approx 0.74$ for all cases where $P / P_{0}=$ 0.56 for Case 1 and $P / P_{0} \approx 0.49$ for other cases. Whereas, after the condensation zone, the slope of the pressure drop of Cases 2-7 accelerates and causes more pressure drop (this is observable in Figs. $8 \mathrm{~b}$ and 8c). Comparing two illustrated contours of the static pressure, it can be stated that existing the wetness at inlet led to more pressure drop in the condensation zone while, conversely, the static pressure increases at the trailing edge. 


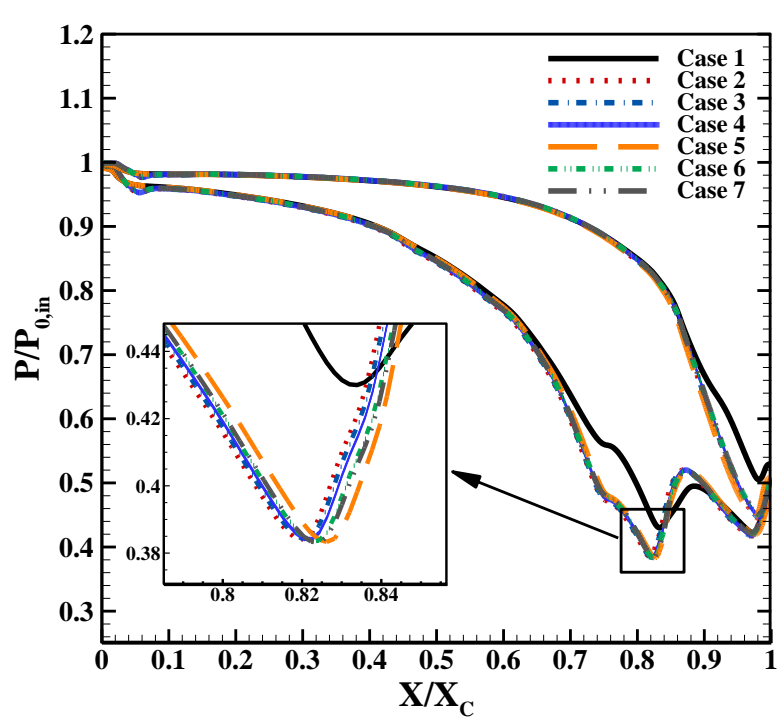

a

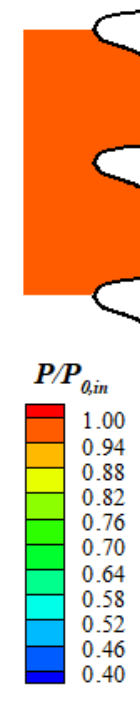

.40

Fig. 8: Static pressure distribution a) in different values of wetness, b) in blade cascade for Case 1, and c) Case 7

The Mach number distributions obtained by the proposed grid are demonstrated in Fig. 9a. As observed, the Mach number increased gradually, and the shock occurred at $x / x_{c} \approx 0.84$ where the Mach number has the maximum value (Fig. 9a). In addition, by comparing Figs. 8a and 9a, it can be stated that despite more pressure drop, Mach number rises due to considering wetness at the inlet. Also, it is an observable lower amount of wetness caused the higher value of Mach number. This means that wet at the inlet can increase the flow velocity and, consequently, the rate of kinetic energy. As a result, the wet-steam flow reaches with a higher amount of kinetic energy to the rotor.

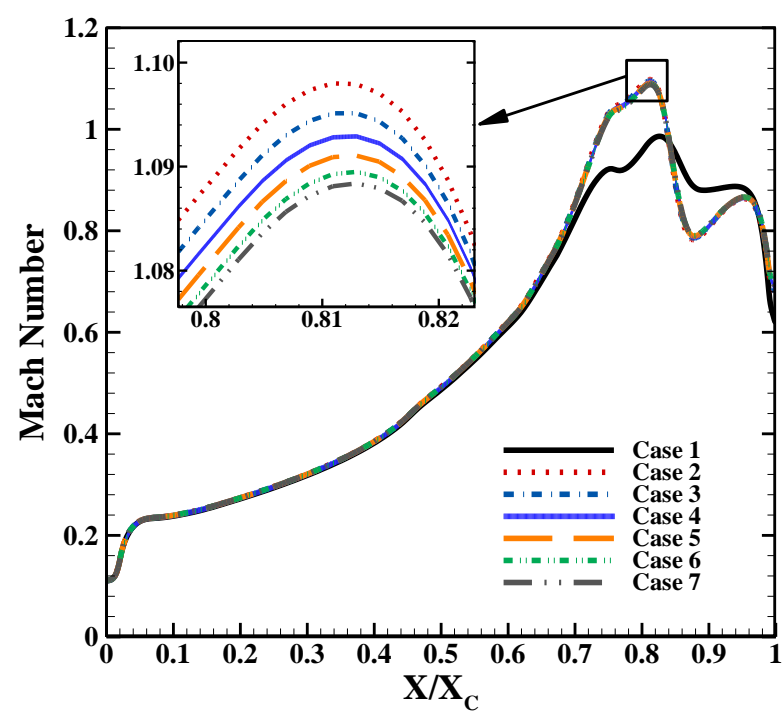

a

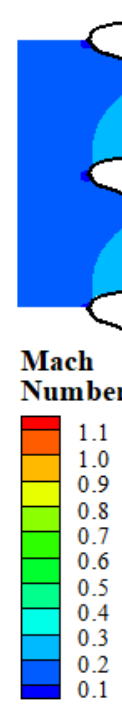

Number

0.1

Fig. 9: Mach Number distribution a) in different values of wetness on suction surface, b) in blade cascade for Case 1, and c) Case 7

Variations in the supercooling degree along the suction surface are represented by Fig. 10a which is defined as the difference between the vapor temperature and the saturation 
temperature corresponding to the pressure of flow $T_{S}(P)-T_{v}$. As can be seen, the diagram of the supercooling degree has an increasing trend until the condensation shock occurs on the suction side at $x / x_{c} \approx 0.74$ and the highest value of the supercooling degree is equal to $33 \mathrm{~K}$ at the location of condensation shock. Afterward, the supercooling degree rapidly drops which is due to a difference in the pressure, and finally, the variation is decreased due to the reduction of the difference between the vapor and the droplets temperatures. In addition, it is clearly observable that existence of slight amount of wetness at inflow, effectively decreases the supercooling degree and consequently deviation from equilibrium condition. Moreover, the location of the highest value of the supercooling degree is moved toward downstream, which shows retarding the nucleation phenomenon. Comparison between two values of wetness represented in Figs. 10b and 10c.

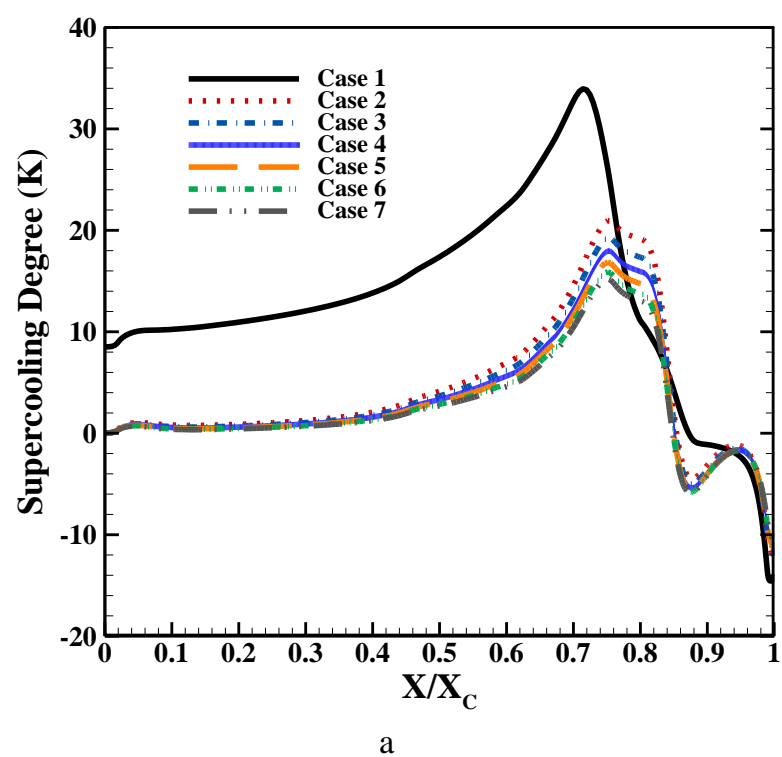

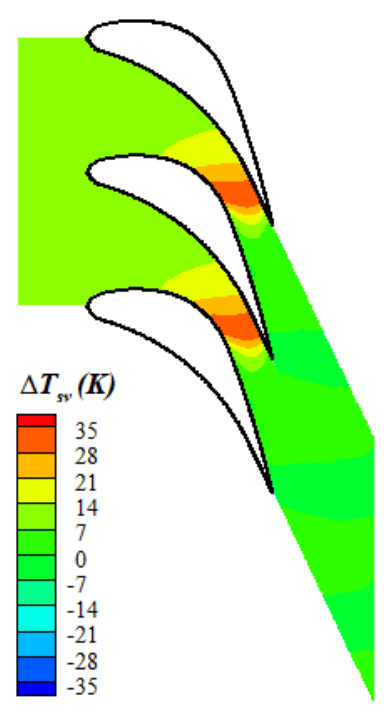

b

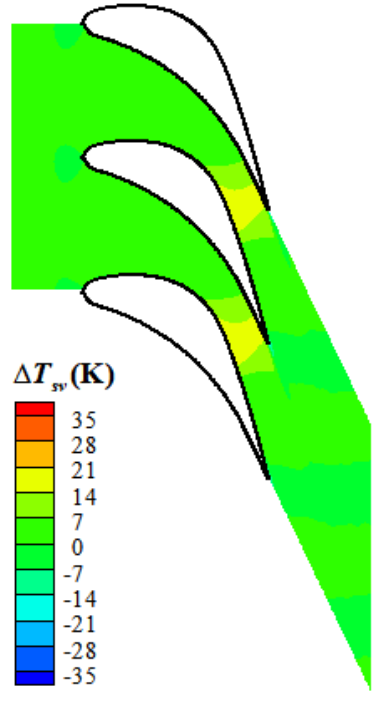

$\mathrm{c}$

Fig. 10: Supercooling degree variation a) in different values of wetness on suction surface, $b$ ) in blade cascade for Case 1, and c) Case 7

Fig. 11a depicts the droplet nucleation rate computed by the proposed NURBS-based grid. As shown, adding wetness to the inflow led to a decrease in the rate of droplet nucleation. For Cases 6 and 7, the rate of nucleation on the suction surface is zero while there is still a slight nucleation throughout the flow in Case 6. Comparing Figs. 11b and 11c, it can be observed that no droplet nucleation has occurred in Case 7.

Distribution of droplet radius on the suction surface is demonstrated by Fig. 12a. As shown, the droplet radius of Case 1 begins to rise when the nucleation rate has the maximum value. Afterward, the droplet growth decreases and reaches the constant value at the outlet. However, no significant growth of droplet can be seen for Cases 2 to 7 and there is a radius decrease for the mentioned cases. In addition, Fig. 12b and 12c show that there is a remarkable difference in droplet size between Cases 1 and 7. 


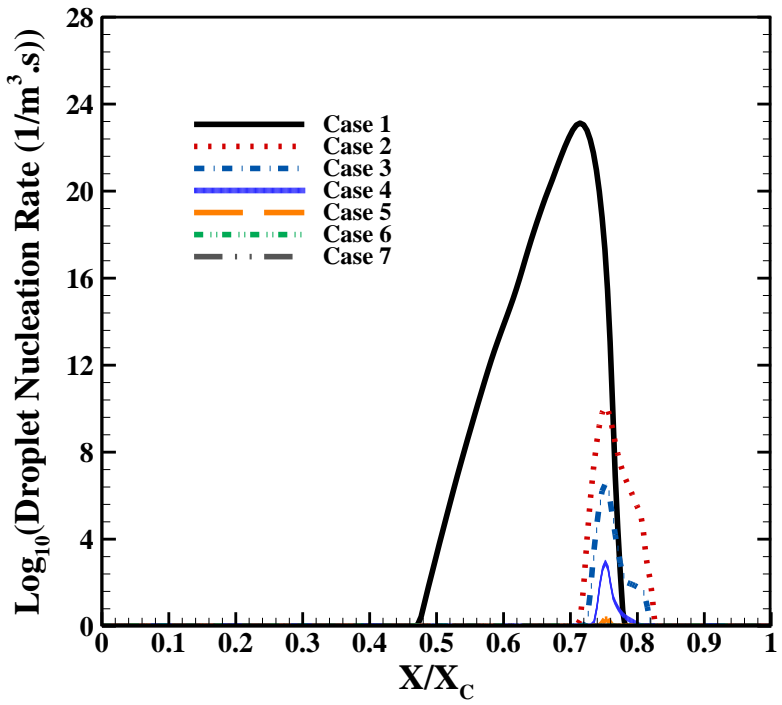

a

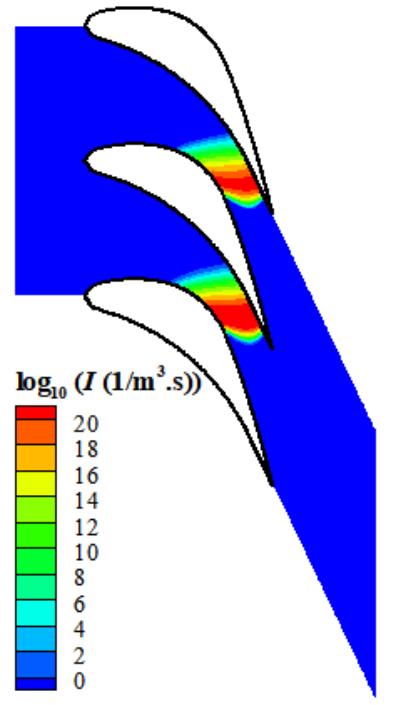

$\mathrm{b}$

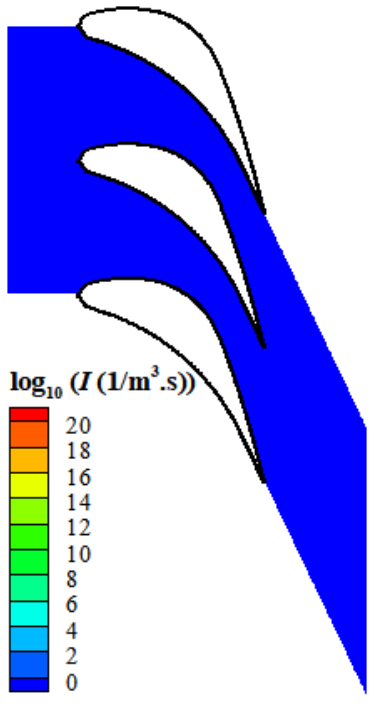

C

Fig. 11: Nucleation rate distribution a) in different values of wetness on suction surface, b) in blade cascade for Case 1, and c) Case 7

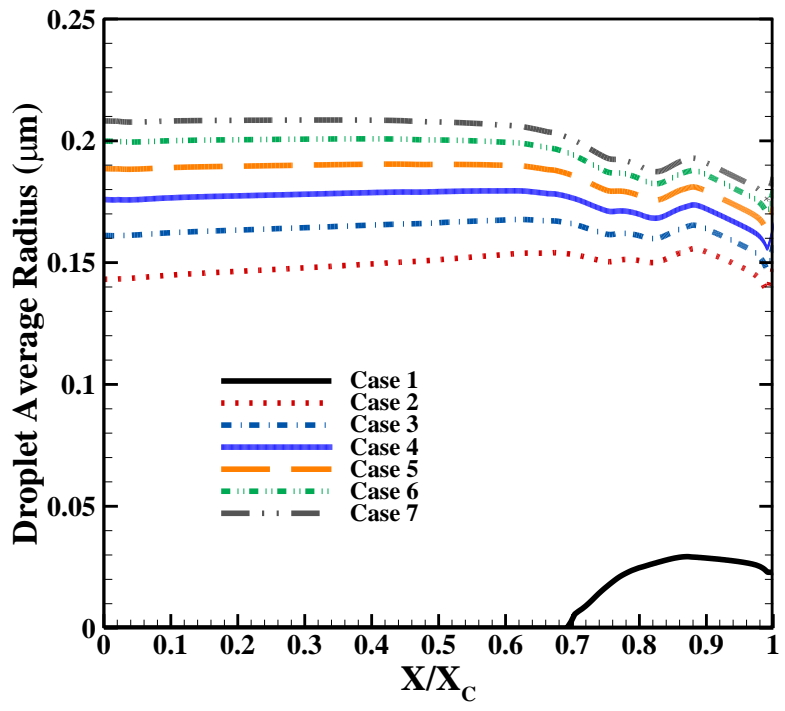

a

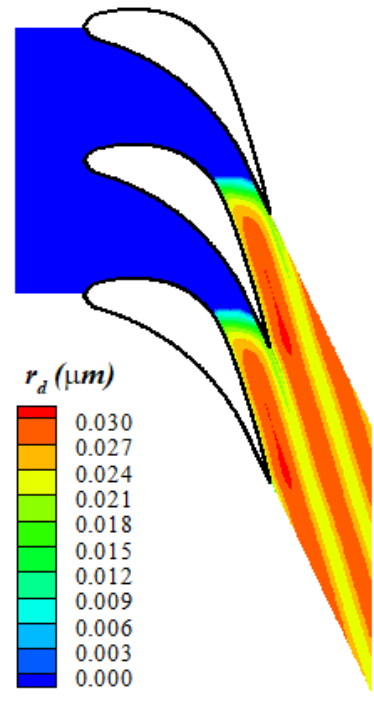

$\mathrm{b}$

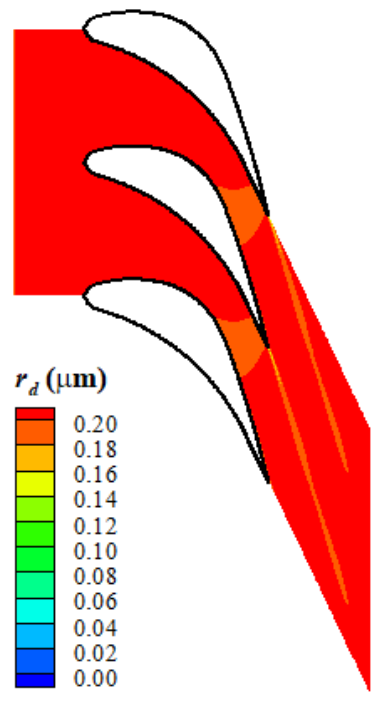

c

Fig. 12: Distribution of droplets radius a) in different values of wetness on suction surface, b) in blade cascade for Case 1, and c) Case 7

The wetness fraction calculated by implementing the NURBS technique is illustrated in Fig. 13. As shown, the wetness fraction is rapidly increased by beginning the nucleation process. Moreover, a decrease in wetness fraction of the flow can be observed which is due to passing the location of aerodynamic shock and also the increase of evaporation temperature. Moreover, the wetness faction increases by increasing the wetness of inflow. It is worth mentioning that existence of higher values of wetness and bigger droplets at inlet can be led to more erosion damage and more decrease in efficiency of the blade. Nevertheless, it cannot be ignored that wet at inlet is able to stop the nucleation process and also deliver a flow with higher kinetic energy to the rotor. 


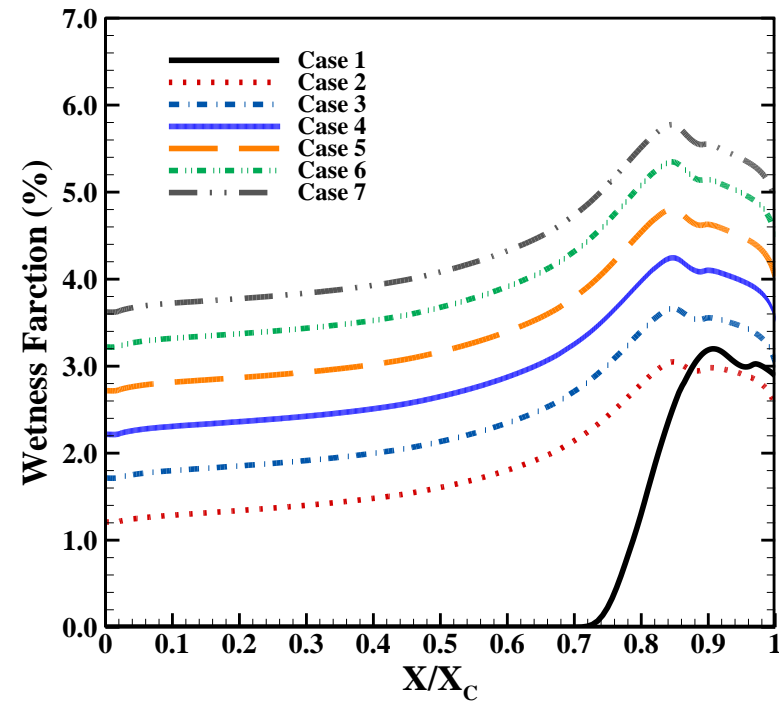

a

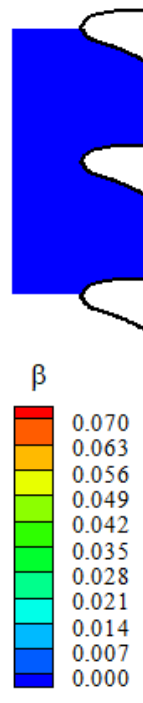

0.000

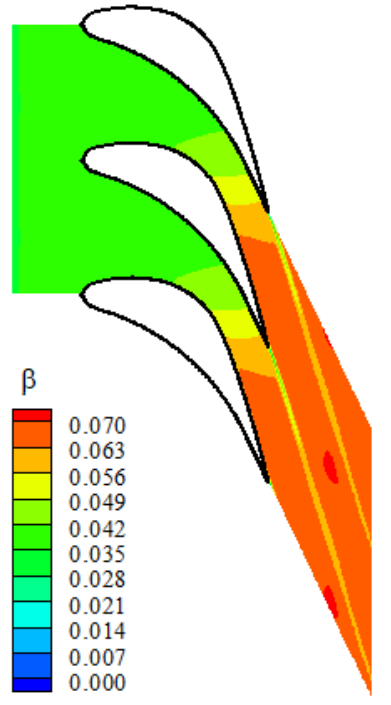

c

Fig. 13: Wetness fraction variation a) in different values of wetness on suction surface, b) in blade cascade for Case 1, and c) Case 7

\section{Conclusion}

In this paper, the effect of existence of wetness at the inlet of steam turbine stationary cascades is investigated. In addition, a NURBS-based technique was used as a new mesh generation method in order to achieve a more compatible grid with the geometry of the blade cascade. In this regard, two different approaches were adopted and the impact of variation of the angle of the grid at the trailing edge was studied. The computed results were also validated against the experimental data and compared to the calculated results of the typical H-type gird. Results indicated that applying the grid generated by the NURBS formulation is well consistent with the experiments and could predict and track the shock locations accurately. Additionally, it was observed that using an angle of the grid equal to $64.4^{\circ}$, generated according to the proposed BB approach, resulted in the good agreement with the experiment. Therefore, the mentioned grid was proposed in order to investigate the wet-steam flow. Compared to the typical H-type grid, the proposed NURBS-based mesh improved the prediction accuracy of static pressure distribution and droplet average radius. Finally, according to the computed results of the wet inflow, it was observed that Mach number rises due to the existence of wetness at inlet. Therefore, the rate of kinetic energy increases because of velocity increase. Additionally, the supercooling degree was effectively decreased which means the deviation from equilibrium condition was reduced. Moreover, it was observed that the nucleation process could be alleviated when we have $3.0 \%$ wetness in the inflow.

\section{Acknowledgment}

Ali Hashemian was partially funded by the BCAM "Severo Ochoa" accreditation of excellence (SEV-2017-0718), the Basque Government through the BERC 2018-2021 program, and the Project of the Spanish Ministry of Science and Innovation with reference PID2019-108111RBI00 (FEDER/AEI). 


\section{References}

[1] Y. Yang and S. Shen, "Numerical simulation on non-equilibrium spontaneous condensation in supersonic steam flow," International Communications in Heat and Mass Transfer, vol. 36, pp. 902-907, 2009.

[2] G. Jiang, J. Gao, and X. Shi, "Flow and heat transfer characteristics of mist/steam twophase flow in the U-shaped cooling passage with $60 \mathrm{deg}$. ribs," International Communications in Heat and Mass Transfer, vol. 105, pp. 73-83, 2019.

[3] A. Gerber and M. Kermani, "A pressure based Eulerian-Eulerian multi-phase model for non-equilibrium condensation in transonic steam flow," International Journal of Heat and Mass Transfer, vol. 47, pp. 2217-2231, 2004.

[4] S. Dykas and W. Wróblewski, "Two-fluid model for prediction of wet steam transonic flow," International Journal of Heat and Mass Transfer, vol. 60, pp. 88-94, 2013.

[5] Y. Patel, G. Patel, and T. Turunen-Saaresti, "Influence of turbulence modelling on nonequilibrium condensing flows in nozzle and turbine cascade," International Journal of Heat and Mass Transfer, vol. 88, pp. 165-180, 2015.

[6] Y. Dai, Y. Cheng, J. Zou, and D. Hu, "Superheating and supercharging characteristics of moving shock in wet steam flows," International Journal of Heat and Mass Transfer, vol. 86, pp. 351-357, 2015.

[7] M. Vatanmakan, E. Lakzian, and M. R. Mahpeykar, "Investigating the entropy generation in condensing steam flow in turbine blades with volumetric heating," Energy, vol. 147, pp. 701-714, 2018.

[8] S. Yamamoto, S. Moriguchi, H. Miyazawa, and T. Furusawa, "Effect of inlet wetness on transonic wet-steam and moist-air flows in turbomachinery," International Journal of Heat and Mass Transfer, vol. 119, pp. 720-732, 2018.

[9] H. Mashmoushy, M. R. Mahpeykar, and F. Bakhtar, "Studies of nucleating and wet steam flows in two-dimensional cascades," Proceedings of the Institution of Mechanical Engineers, Part C: Journal of Mechanical Engineering Science, vol. 218, pp. 843-858, 2004.

[10] A. H. Yousif, A. M. Al-Dabagh, and R. C. Al-Zuhairy, "Non-equilibrium spontaneous condensation in transonic steam flow," International Journal of Thermal Sciences, vol. 68, pp. 32-41, 2013.

[11] S. Dykas, M. Majkut, M. Strozik, and K. Smołka, "Experimental study of condensing steam flow in nozzles and linear blade cascade," International Journal of Heat and Mass Transfer, vol. 80, pp. 50-57, 2015.

[12] S. Dykas, M. Majkut, K. Smołka, and M. Strozik, "Study of the wet steam flow in the blade tip rotor linear blade cascade," International Journal of Heat and Mass Transfer, vol. 120, pp. 9-17, 2018.

[13] W. Wan, K. Engvall, W. Yang, and B. F. Möller, "Experimental and modelling studies on condensation of inorganic species during cooling of product gas from pressurized biomass fluidized bed gasification," Energy, vol. 153, pp. 35-44, 2018.

[14] R. Hagmeijer, R. H. IJzermans, and F. Put, "Solution of the general dynamic equation along approximate fluid trajectories generated by the method of moments," Physics of fluids, vol. 17, p. 056101, 2005.

[15] S. Sinha, B. E. Wyslouzil, and G. Wilemski, "Modeling of H2O/D2O condensation in supersonic nozzles," Aerosol Science and Technology, vol. 43, pp. 9-24, 2009.

[16] J. Young, "The spontaneous condensation of steam in supersonic nozzle," Physico Chemical Hydrodynamics, vol. 3, pp. 57-82, 1982.

[17] F. Bakhtar and K. Zidi, "On the self diffusion of water vapour," Proceedings of the Institution of Mechanical Engineers, Part C: Journal of Mechanical Engineering Science, vol. 199, pp. 159-164, 1985. 
[18] E. Lakzian and A. Masjedi, "Slip effects on the exergy loss due to irreversible heat transfer in a condensing flow," International Journal of Exergy, vol. 14, pp. 22-37, 2014.

[19] E. Lakzian and S. Shaabani, "Analytical investigation of coalescence effects on the exergy loss in a spontaneously condensing wet-steam flow," International Journal of Exergy, vol. 16, pp. 383-403, 2015.

[20] N. Sakai, T. Harada, and Y. Imai, "Numerical study of partial admission stages in steam turbine," JSME International Journal Series B Fluids and Thermal Engineering, vol. 49, pp. 212-217, 2006.

[21] L. Zhang, L. He, and H. Stuer, "A numerical investigation of rotating instability in steam turbine last stage," in ASME 2011 Turbo Expo: Turbine Technical Conference and Exposition, 2011, pp. 1657-1666.

[22] J. Joseph, S. Subramanian, K. Vigney, B. Prasad, and D. Biswas, "Thermodynamic wetness loss calculation in nozzle and turbine cascade: nucleating steam flow," Heat and Mass Transfer, pp. 1-11, 2017.

[23] F. Bakhtar, M. R. Mahpeykar, and K. Abbas, "An investigation of nucleating flows of steam in a cascade of turbine blading-theoretical treatment," Journal of fluids engineering, vol. 117, pp. 138-144, 1995.

[24] F. Bakhtar, M. Zamri, and J. Rodriguez-Lelis, "A comparative study of treatment of two-dimensional two-phase flows of steam by a Runge-Kutta and by Denton's methods," Proceedings of the Institution of Mechanical Engineers, Part C: Journal of Mechanical Engineering Science, vol. 221, pp. 689-706, 2007.

[25] F. Bakhtar and M. M. Tochai, "An investigation of two-dimensional flows of nucleating and wet steam by the time-marching method," International Journal of Heat and Fluid Flow, vol. 2, pp. 5-18, 1980.

[26] M. S. Mirhoseini and M. Boroomand, "Multi-objective optimization of hot steam injection variables to control wetness parameters of steam flow within nozzles," Energy, vol. 141, pp. 1027-1037, 2017.

[27] G. Francesco, M. Federico, and M. Adriano, "CFD modelling of the condensation inside a cascade of steam turbine blades: comparison with an experimental test case," Energy Procedia, vol. 126, pp. 730-737, 2017.

[28] A. Hashemian, S. F. Hosseini, and S. N. Nabavi, "Kinematically Smoothing Trajectories by NURBS Reparameterization - An Innovative Approach," Advanced Robotics, vol. 31, pp. 1296-1312, 2017.

[29] A. J. Herrema, N. M. Wiese, C. N. Darling, B. Ganapathysubramanian, A. Krishnamurthy, and M.-C. Hsu, "A framework for parametric design optimization using isogeometric analysis," Computer Methods in Applied Mechanics and Engineering, vol. 316, pp. 944-965, 2017.

[30] N. Nguyen-Thanh, K. Zhou, X. Zhuang, P. Areias, H. Nguyen-Xuan, Y. Bazilevs, et al., "Isogeometric analysis of large-deformation thin shells using RHT-splines for multiple-patch coupling," Computer Methods in Applied Mechanics and Engineering, vol. 316, pp. 1157-1178, 2017.

[31] A. Hashemian and S. F. Hosseini, "Nonlinear bifurcation analysis of statically loaded free-form curved beams using isogeometric framework and pseudo-arclength continuation," International Journal of Non-Linear Mechanics, vol. 113, pp. 1-16, 2019.

[32] P. Bo and M. Bartoň, "On initialization of milling paths for 5-axis flank CNC machining of free-form surfaces with general milling tools," Computer Aided Geometric Design, vol. 71, pp. 30-42, 2019. 
[33] A. Hashemian, P. Bo, and M. Bartoň, "Reparameterization of Ruled Surfaces: Toward Generating Smooth Jerk-minimized Toolpaths for Multi-axis Flank CNC Milling," Computer-Aided Design, vol. 127, p. 102868, 2020.

[34] Y. Bazilevs and T. Hughes, "NURBS-based isogeometric analysis for the computation of flows about rotating components," Computational Mechanics, vol. 43, pp. 143-150, 2008.

[35] M. T. Herath, S. Natarajan, B. G. Prusty, and N. S. John, "Isogeometric analysis and Genetic Algorithm for shape-adaptive composite marine propellers," Computer Methods in Applied Mechanics and Engineering, vol. 284, pp. 835-860, 2015.

[36] S. Senoo, K. Ogata, T. Nakamura, and N. Shibashita, "Three-dimensional design method for long blades of steam turbines using fourth-degree nurbs surface," in ASME Turbo Expo 2010: Power for Land, Sea, and Air, 2010, pp. 2149-2158.

[37] T. Tejima, Y. Niizeki, N. Shibukawa, A. Onoda, S. Watanabe, X. Yuan, et al., "Three dimensional optimum design of a steam turbine stage with nurbs curves," in ASMEJSME-KSME 2011 Joint Fluids Engineering Conference, 2011, pp. 1773-1783.

[38] P. Stein, M.-C. Hsu, Y. Bazilevs, and K. Beucke, "Operator-and template-based modeling of solid geometry for Isogeometric Analysis with application to Vertical Axis Wind Turbine simulation," Computer Methods in Applied Mechanics and Engineering, vol. 213, pp. 71-83, 2012.

[39] A. M. Abbass, M. M. Sorour, and M. A. Teamah, "Evaluation of Different Turbulence Models and Numerical Solvers for a Transonic Turbine Blade Cascade," Journal of Energy and Power Sources, vol. 1, pp. 17-30, 2014.

[40] K. Nordanger, R. Holdahl, T. Kvamsdal, A. M. Kvarving, and A. Rasheed, "Simulation of airflow past a 2D NACA0015 airfoil using an isogeometric incompressible NavierStokes solver with the Spalart-Allmaras turbulence model," Computer Methods in Applied Mechanics and Engineering, vol. 290, pp. 183-208, 2015.

[41] A. Hashemian and S. F. Hosseini, "An integrated fitting and fairing approach for object reconstruction using smooth NURBS curves and surfaces," Computers \& Mathematics with Applications, vol. 76, pp. 1555-1575, 2018.

[42] W. G. Courtney, "Remarks on homogeneous nucleation," The Journal of Chemical Physics, vol. 35, pp. 2249-2250, 1961.

[43] A. Kantrowitz, "Nucleation in very rapid vapor expansions," The Journal of Chemical physics, vol. 19, pp. 1097-1100, 1951.

[44] J. Young, "Two-dimensional, nonequilibrium, wet-steam calculations for nozzles and turbine cascades," Journal of Turbomachinery, vol. 114, pp. 569-579, 1992.

[45] A. Ahmadpour, S. M. A. Noori Rahim Abadi, and J. P. Meyer, "On the performance enhancement of thermo-compressor and steam turbine blade cascade in the presence of spontaneous nucleation," Energy, vol. 119, pp. 675-693, 2017.

[46] H. Bagheri Esfe, M. J. Kermani, and M. Saffar Avval, "Effects of surface roughness on deviation angle and performance losses in wet steam turbines," Applied Thermal Engineering, vol. 90, pp. 158-173, 2015.

[47] A. R. Avetissian, G. A. Philippov, and L. I. Zaichik, "Effects of turbulence and inlet moisture on two-phase spontaneously condensing flows in transonic nozzles," International Journal of Heat and Mass Transfer, vol. 51, pp. 4195-4203, 2008.

[48] S. Yamamoto, "Computation of practical flow problems with release of latent heat," Energy, vol. 30, pp. 197-208, 2005.

[49] K. Ishizaka, "A high-resolution numerical method for transonic non-equilibrium condensation flow through a steam turbine cascade," Proc. of the 6th ISCFD, 1995, vol. 1, pp. 479-484, 1995. 
[50] X.-D. Wang, J.-L. Dong, T. Wang, and J.-Y. Tu, "Numerical analysis of spontaneously condensing phenomena in nozzle of steam-jet vacuum pump," Vacuum, vol. 86, pp. 861-866, 2012.

[51] F. Bakhtar and M. R. Mahpeykar, "On the performance of a cascade of turbine rotor tip section blading in nucleating steam Part 3: theoretical treatment," Proceedings of the Institution of Mechanical Engineers, Part C: Journal of Mechanical Engineering Science, vol. 211, pp. 195-210, 1997.

[52] F. Bakhtar, J. Young, A. White, and D. Simpson, "Classical nucleation theory and its application to condensing steam flow calculations," Proceedings of the Institution of Mechanical Engineers, Part C: Journal of Mechanical Engineering Science, vol. 219, pp. 1315-1333, 2005.

[53] M. Kermani and A. Gerber, "A general formula for the evaluation of thermodynamic and aerodynamic losses in nucleating steam flow," International journal of heat and mass transfer, vol. 46, pp. 3265-3278, 2003.

[54] B. Nikkhahi, M. Shams, and M. Ziabasharhagh, "A numerical investigation of twophase steam flow around a 2-D turbine's rotor tip," International Communications in Heat and Mass Transfer, vol. 36, pp. 632-639, 2009.

[55] J. Young, "An equation of state for steam for turbomachinery and other flow calculations," Journal of engineering for gas turbines and power, vol. 110, pp. 1-7, 1988.

[56] F. R. Menter, "Two-equation eddy-viscosity turbulence models for engineering applications," AIAA journal, vol. 32, pp. 1598-1605, 1994.

[57] Y. Yang, C. Wen, S. Wang, and Y. Feng, "Effect of inlet and outlet flow conditions on natural gas parameters in supersonic separation process," PloS one, vol. 9, p. e110313, 2014.

[58] M. Federico, G. Francesco, and M. Adriano, "CFD modelling of the condensation inside a Supersonic Nozzle: implementing customized wet-steam model in commercial codes," Energy Procedia, vol. 126, pp. 34-41, 2017.

[59] L. Piegl and W. Tiller, The NURBS Book, 2nd ed. New York, NY: Springer-Verlag, 1997.

[60] P. L. Roe, "Characteristic-based schemes for the Euler equations," Annual review of fluid mechanics, vol. 18, pp. 337-365, 1986.

[61] D. Holmes and S. Connell, "Solution of the 2D Navier-Stokes equations on unstructured adaptive grids," in 9th Computational Fluid Dynamics Conference, 1989, p. 1932.

[62] A. S. Liberson and S. H. Hesler, "A Unified CFD Based Approach to a Variety of Condensation Processes in a Viscous Turbulent Wet Steam Flow," International Journal of Modern Nonlinear Theory and Application, vol. 6, p. 85, 2017.

[63] A. Rueda, M. Toledo, F. Rueda, and R. Rangel, "Numerical Investigation of the Droplet Behavior in Cascades Using a Finite Volume Method," Computación y Sistemas, vol. 21, pp. 473-484, 2017.

[64] A. J. White, J. B. Young, and P. T. Walters, "Experimental validation of condensing flow theory for a stationary cascade of steam turbine blades," Philosophical Transactions of the Royal Society of London. Series A: Mathematical, Physical and Engineering Sciences, vol. 354, pp. 59-88, 1996. 